\title{
On the desert between neutron star and black hole remnants
}

\author{
R. Caimmi*
}

January 8, 2019

\begin{abstract}
The occurrence of a desert between neutron star (NS) and black hole $(\mathrm{BH})$ remnants is reviewed using a set of well-determined masses from different sources. The dependence of stellar remnants on the zero age main sequence (ZAMS) progenitor mass for solar metallicity is taken from a recent investigation and further effort is devoted to NS and $\mathrm{BH}$ remnants. In particular, a density parameter is defined and related to NS mass and radius. Spinning BHs in Kerr metrics are considered as infinitely thin, homogeneous, rigidly rotating disks in Newtonian mechanics. Physical parameters for nonrotating (TOV) and equatorial breakup (EQB) configurations are taken or inferred from a recent investigation with regard to $4 \mathrm{NS}$ and 3 quark star (QS) physically motivated equation of state (EOS) kinds. A comparison is performed with counterparts related to nonrotating and maximally rotating BHs. The results are also considered in the light of empirical relations present in literature. With regard to $J-M$ relation, EQB configurations are placed on a sequence of similar slope in comparison to maximally rotating $\mathrm{BHs}$, but shifted downwards due to lower angular momentum by a factor of about 3.6. Under the assumption heavy baryons are NS constituents and instantaneously undergo quark-level
\end{abstract}

*Physics and Astronomy Department, Padua University, Vicolo Osservatorio 3/2, I-35122 Padova, Italy. Affiliated up to September 30th 2014. Current status: Studioso Senior. Current position: in retirement due to age limits. email: roberto.caimmi@unipd.it fax: 39-049-8278212 
reactions, the energy released (or adsorbed) is calculated using results from a recent investigation. Even if NSs exclusively host heavy baryons of the kind considered, the total amount cannot exceed about $10 \%$ of the binding energy, which inhibits supernova explosions as in supramassive white dwarf (WD) remnants or implosions into BH. Alternative channels for submassive $\left(2 \lesssim M / m_{\odot} \lesssim 4\right)$ BH formation are shortly discussed. Whether the desert between NS and BH remnants could be ascribed to biases and/or selection effects, or related to lack of formation channels, still remains an open question. To this respect, increasingly refined theoretical and observational techniques are needed.

keywords - stars: evolution - stellar remnants - nucleosynthesis neutron stars - black holes.

\section{Introduction}

Stellar remnants are ending configurations (if any) of related zero-age main sequence (ZAMS) progenitors at the end of stellar evolution. Leaving aside brown dwarf (BD) stars, which might be considered as ending configurations by themselves after a short period of D- and possibly Li-burning [103], a stellar remnant can be classified as white dwarf (WD), neutron star (NS) including hybrid stars and quark stars (QS), black hole (BH).

WD progenitors are characterized by sufficiently low masses where nuclear reactions are inhibited before Fe nucleosynthesis. More specifically, ZAMS progenitors within the mass range, $0.5 \lesssim M / m_{\odot} \lesssim 8$, end stellar evolution as carbon-oxygen WDs. Nonrotating WDs are unstable above the Chandrasekhar mass limit, $M_{\mathrm{Ch}} \approx 1.44 m_{\odot}$, yielding a supernova explosion of type Ia e.g., [10]. Ultramassive WDs could occur in presence of fast rotation e.g., [75] [76] or strong magnetic fields e.g., [53] [26].

NS progenitors are characterized by sufficiently large masses where nuclear reactions allow Fe nucleosynthesis. More specifically, ZAMS progenitors within the range, $8 \lesssim M / m_{\odot} \lesssim 120$, end stellar evolution as either NSs after core collapse and supernova explosion of type II, or BHs. Nonrotating NSs are unstable above a threshold, $M_{\mathrm{TOV}} \gtrsim 2 m_{\odot}$, depending on nuclear matter equation of state (EOS) e.g., [58] [117] [78] [97] [118] [90] [98] [102]. Ultramassive NSs could occur in presence of fast rotation e.g., [47] [96] [123] or strong magnetic fields e.g., 63].

$\mathrm{BH}$ progenitors lack a clear mass distinction with respect to NS progenitors. According to a recent investigation [93], the above mentioned ZAMS progenitors co-exist within the mass range, $15 \lesssim M / m_{\odot} \lesssim 21$; 
$25 \lesssim M / m_{\odot} \lesssim 28 ; 60 \lesssim M / m_{\odot} \lesssim 120 ;$ while only the former takes place for $10 \lesssim M / m_{\odot} \lesssim 15$ and the only latter for $21 \lesssim M / m_{\odot} \lesssim 25$; $28 \lesssim M / m_{\odot} \lesssim 60$. The occurrence of NS or BH remnants might be related to different extents of mass loss during evolution, with the addition of neutrino transport and multidimensional turbolence during collapse e.g., [93].

The transition from WD to NS remnants is continuous and exhibits overlapping, in the sense that NS masses occasionally fall below Chandrasekhar mass e.g., [78]. Conversely, the transition from NS to BH remnants shows a desert within the mass range, $2 \lesssim M / m_{\odot} \lesssim 4$ e.g., [31]. In fact, low-mass BH remnants exceed $4 m_{\odot}$ e.g., [40] [42]. Concerning X-ray transients with low-mass donors, it has been argued that understimates of the inclination, by $10^{\circ}$ at least, could significantly reduce $\mathrm{BH}$ mass estimates, filling the gap between the low end of $\mathrm{BH}$ mass distribution and the maximum theoretical NS mass [52].

Submassive $\left(2 \lesssim M / m_{\odot} \lesssim 4\right)$ BH remnants could be the product of WDWD, WD-NS, NS-NS mergers after orbital decay via gravitational radiation as in the recent event GW 170817, where the inferred mass amounts to $2.73_{-0.01}^{+0.04} m_{\odot}$ [2]. With regard to merger product, $\mathrm{BH}$ is largely favoured but ultramassive NS exhibiting a millisecond spin period cannot still be excluded e.g., [4].

Models of stellar evolution could occasionally yield submassive BH remnants after significant amount of fallback from supernovae. If otherwise, the lower $\mathrm{BH}$ mass limit from computations reads $\left(M_{\mathrm{BH}}\right)_{\min } \approx 4 m_{\odot}$, which is related to the He-core mass of ZAMS progenitors where $M_{\mathrm{ZAMS}}=15 \mathrm{~m}_{\odot}$ for $Z=Z_{\odot}$ 93.

The rarity of the above mentioned events, i.e. orbital decay in binary systems hosting WDs and/or NSs, and BHs following fallback from supernovae, by itself provides an explanation to the desert between NS and $\mathrm{BH}$ remnants.

In this view, a nontrivial question reads: "Could ultramassive NSs, or in general putative $\mathrm{BH}$ progenitors, end their life via supernova explosion similarly to supramassive $\left(M \gtrsim 1.44 m_{\odot}\right)$ WDs?" Ultramassive NSs and high-density i.e. low-mass putative $\mathrm{BH}$ progenitors are expected to host, among others, bottomed and/or charmed heavy baryons e.g., [88]. According to a recent investigation, the above mentioned heavy baryons can undergo quark-level fusion to release energy [50], see also [69].

A desert within the mass range, $2 \lesssim M / m_{\odot} \lesssim 4$, would naturally occur if NS remnants can undergo supernova explosion leaving no remnant. If otherwise, different channels should be taken into considerations. The current 
paper aims to investigate what is the case. Stellar remnants exhibiting well determined masses are presented in Section 2. Considerations about NSs are made in Section 3, where attention is focused on a density parameter related to neutron mean density. Considerations about BHs are made in Section 4. where attention is focused on spin parameter, description in terms of homogeneous, infinitely thin, rigidly rotating disks, and comparison between non rotating and maximally rotating $\mathrm{BH}$ and NS/QS configurations. An upper limit to the energy released via quark-level fusion in heavy baryons is estimated in Section 5. The discussion is performed in Section 6. The conclusion is presented in Section 7 .

\section{Stellar remnants}

Stellar remnants are the final product of stellar evolution, where "star" means some kind of nucleosynthesis has occurred, to an extent depending on ZAMS progenitor mass.

Low-mass $\left(0.012 \lesssim M / m_{\odot} \lesssim 0.06\right)$ BDs can perform D-burning, while high-mass $\left(0.06 \lesssim M / m_{\odot} \lesssim 0.07\right)$ BDs, in addition, Li-burning. After D and $\mathrm{Li}$ exhaustion, an inert $\mathrm{BD}$ can be conceived as a remnant in the aforementioned sense. For further details, an interested reader is addressed to review papers e.g., [17] [22] or research articles e.g., [20] [21] [103] [12] [19].

With regard to formation via gravitational instability vs core accretion, $\mathrm{BD}$ lower mass limit is reduced to about $10 M_{\mathrm{J}}=0.009546 m_{\odot}$ regardless of D-burning e.g., [100]. Accordingly, the above mentioned value shall be assumed as a threshold between BDs and giant planets. Keeping in mind ultramassive BDs could exist [36], BD upper mass limit shall be assumed as coinciding with H-burning limit, $M_{\mathrm{H}}=0.071 m_{\odot}$ e.g., [17] [36].

Low-mass $\left(0.07 \lesssim M / m_{\odot} \lesssim 8\right)$ main sequence (MS) stars can synthesize $\mathrm{H}, \mathrm{He}$, metals up to Mn, according to related ZAMS masses, ending their life as WDs. For nonrotating configurations where Chandrasekhar mass is exceeded, the electron Fermi pressure is no longer able to sustain WDs, which undergo supernova explosions of type Ia. Ultramassive WDs could be preserved by fast differential rotation e.g., [75] [76] or strong magnetic fields e.g., [53] [26].

Large-mass $\left(8 \lesssim M / m_{\odot} \lesssim 120\right)$ MS stars can synthesize all elements up to Fe, ending their life as either NS after core-collapse and supernova explosion of type II, or BH. For sufficiently low masses, core collapse can be halted by Fermi pressure and the result is a NS. If otherwise, gravitational collapse is inescapable yielding a $\mathrm{BH}$.

According to recent investigations, the final fate of massive $\left(M \gtrsim 15 m_{\odot}\right)$ 
star is decided by different processes, such as mass loss and onset of URCA reactions. The result is $\mathrm{NS}$ and $\mathrm{BH}$ co-existence or mutual exclusion in different mass ranges. With regard to solar metallicities, the following NS fraction for assigned ZAMS progenitor mass range has been inferred from numerical simulations [93]:

$$
X_{\mathrm{NS}}= \begin{cases}1 ; & 10 \leq M_{\mathrm{ZAMS}} / m_{\odot} \leq 15 ; \\ 0.6 ; & 15 \leq M_{\mathrm{ZAMS}} / m_{\odot} \leq 21 ; \\ 0 ; & 21 \leq M_{\mathrm{ZAMS}} / m_{\odot} \leq 25 ; \\ 0.75 ; & 25 \leq M_{\mathrm{ZAMS}} / m_{\odot} \leq 28 \\ 0 ; & 28 \leq M_{\mathrm{ZAMS}} / m_{\odot} \leq 60 ; \\ 0.4 ; & 60 \leq M_{\mathrm{ZAMS}} / m_{\odot} \leq 120\end{cases}
$$

where related $\mathrm{BH}$ fraction is $X_{\mathrm{BH}}=1-X_{\mathrm{NS}}$.

Leaving aside fast rotation and strong magnetic fields, NS maximum mass is slightly larger than $2 m_{\odot}$ e.g., [5] [96. Conversely, BH masses from stellar implosions are bounded by He-core and final pre-supernova masses of the progenitor, according to the fraction of stellar envelope which is ejected during or prior to implosion. Related masses depend on the ZAMS progenitor mass as [93]:

$$
\begin{aligned}
& y=-2.049+0.4140 x ; \quad y=M_{\mathrm{BH}, \text { core }} ; \quad x=M_{\mathrm{ZAMS}} ; \\
& y=15.52-0.3294 x-0.02121 x^{2}+0.003120 x^{3} ; \\
& y=M_{\mathrm{BH}, \text { all }} ; \quad x=M_{\mathrm{ZAMS}}-25.97 ;
\end{aligned}
$$

for $15 \leq M_{\mathrm{ZAMS}} / m_{\odot} \leq 40$, where envelope mass of pre-supernova progenitors cannot be neglected with respect to He-core mass, and:

$$
y=5.697+7.8598 \cdot 10^{8} x^{-4.858} ; \quad y=M_{\mathrm{BH}, \text { core }} ; \quad x=M_{\mathrm{ZAMS}} ;
$$

for $45 \leq M_{\mathrm{ZAMS}} / m_{\odot} \leq 120$, where envelope mass of pre-supernova progenitors can safely be neglected with respect to He-core mass. Occasionally, subHe-core (i.e. below He-core mass; in particular, submassive) BH remnants can be formed from massive progenitors via fallback from supernovae [93.

Stellar remnants with well determined masses i.e. percent error $\Delta^{\%} M=$ $100(\Delta M / M)<10$ or $\Delta M / M<0.1$ are shown in Fig.1 as $\log M$ vs $\log M$. Owing to the paucity of known $\mathrm{BH}$ remnants at present, data affected by larger percent error are also included. To avoid confusion, errors are represented as horizontal bars instead of squares. BD, WD, NS, BH points are shown as triangles, squares, diamonds, crosses, respectively. Related values are listed in Appendix $\mathrm{A}$. The post-merger product from the double NS 
binary GW 170817 is shown as a saltire. D-burning limit, assumed to occur at $13 M_{\mathrm{J}}=0.01241 m_{\odot} ; \mathrm{H}$-burning limit, assumed to occur at $0.071 m_{\odot}$; Chandrasekhar mass, assumed to be $1.44 \mathrm{~m}_{\odot}$; minimum He-core mass of $\mathrm{BH}$ progenitors, inferred from numerical simulations as $4 m_{\odot}$ [93]; are marked as vertical lines.

An inspection of Fig. 1 discloses a continuous transition in mass passing from WDs to NSs, where the two domains partially overlap. The gap between BDs and WDs, $0.07 \lesssim M / m_{\odot} \lesssim 0.16$, implies related WD progenitors are still alive as MS dwarfs, also plotted as asterisks and listed in Appendix A. The gap between NSs and BHs could be interpreted as due to selection effects and/or lack of data for massive $\left(M \geq 15 m_{\odot}\right)$ ZAMS progenitors, yielding Hecore masses above $4 m_{\odot}[93]$. Conversely the mass range, $2 \lesssim M / m_{\odot} \lesssim 4$, needs alternative explanations.

To this respect, a viable possibility could be compact remnant i.e. WDWD, WD-NS, NS-NS, merging as recently observed for the double NS pair GW 170817, where the total mass amounts to $2.73_{-0.01}^{+0.04} m_{\odot}$ including ejected matter [2] 4]. Accordingly, the merger product is an isolated $\mathrm{BH}$ in absence of additional system memberships, which could explain the presence of a desert in the above mentioned mass range, keeping in mind isolated BHs are difficult to be detected.

On the other hand, binary systems hosting a NS and a donor MS star could yield a $\mathrm{BH}$ via mass accretion onto NS and subsequent instability, where the presence of a $\mathrm{BH}$ should be inferred as mass accretion goes on. But low-mass BHs in binary systems have not been detected up today. Then a legitimate question is: "Could unstable NSs undergo supernova explosions leaving no remnant?"

\section{About neutron stars}

NS masses typically lie within the range, $1 \lesssim M / m_{\odot} \lesssim 2$, as can be recognized from Fig.11 and Table 12. NS radii typically lie within the range, $9 \lesssim R / \mathrm{km} \lesssim 12$, as can be inferred from the $M$ - $R$ relation assuming reliable EOSs e.g., [78]. Then related mean density is bounded as:

$$
\begin{aligned}
& \bar{\rho}_{\text {min }} \lesssim \bar{\rho} \lesssim \bar{\rho}_{\text {max }} ; \\
& \bar{\rho}_{\text {min }}=\frac{m_{\odot}}{4 \pi(12 \mathrm{~km})^{3} / 3}=2.747288 \cdot 10^{17} \frac{\mathrm{kg}}{\mathrm{m}^{3}} \\
& \bar{\rho}_{\max }=\frac{2 m_{\odot}}{4 \pi(9 \mathrm{~km})^{3} / 3}=1.302418 \cdot 10^{18} \frac{\mathrm{kg}}{\mathrm{m}^{3}}
\end{aligned}
$$


to be compared with nuclear saturation density, $\rho_{\text {sat }} \approx 2.8 \cdot 10^{17} \mathrm{~kg} / \mathrm{m}^{3}$ e.g., [78] [47.

With regard to neutrons in atomic nuclei (intended in ordinary conditions), mass and radius shall be assumed as $m_{\mathrm{n}}=1.675 \cdot 10^{-27} \mathrm{~kg}$ and $r_{\mathrm{n}}=10^{-15} \mathrm{~m}$, respectively, yielding the following neutron mean density:

$$
\bar{\rho}_{\mathrm{n}}=\frac{3}{4 \pi} \frac{m_{\mathrm{n}}}{r_{\mathrm{n}}^{3}}=3.9988 \cdot 10^{17} \frac{\mathrm{kg}}{\mathrm{m}^{3}} ;
$$

which is comparable to NS mean density and, for this reason, shall be taken as a reference density. On the other hand, a recent investigation has disclosed central pressure inside protons is larger than inside NSs [18].

The ratio:

$$
\zeta_{\mathrm{n}}=\frac{\bar{\rho}_{\mathrm{n}}}{\bar{\rho}}=\frac{m_{\mathrm{n}}}{M} \frac{R^{3}}{r_{\mathrm{n}}^{3}}
$$

may be intended as a density factor, where $\zeta_{n}>1$ implies lower NS mean density with respect to neutron and the contrary holds for $\zeta_{\mathrm{n}}<1$.

In explicit form, Eq. (7) reads:

$$
\zeta_{\mathrm{n}}=\frac{m_{\mathrm{n}}}{m_{\odot}} \frac{m_{\odot}}{M} \frac{R^{3}}{\mathrm{~km}^{3}} \frac{\mathrm{km}^{3}}{r_{\mathrm{n}}^{3}}=0.8423223 \frac{(R / \mathrm{km})^{3}}{M / m_{\odot}} ;
$$

where mass and radius are expressed in solar masses and kilometers, respectively.

NS density factor, $\zeta_{n}$, vs radius, $R / \mathrm{km}$, for assigned mass, $M / m_{\odot}$, is plotted in Fig.2, where most of currently available or inferred data e.g., [78] lie within a region bounded by $R / \mathrm{km}=9,12$, and $M / m_{\odot}=1$, 2, e.g., [78], which implies $0.3 \lesssim \zeta_{\mathrm{n}} \lesssim 1.5$.

NS mass, $M / m_{\odot}$, vs radius, $R / \mathrm{km}$, for assigned density factor, $\zeta_{\mathrm{n}}$, is plotted in Fig. 3, where the rectangular area is the counterpart of the region shown in Fig.2,

The gravitational i.e. Schwartzschild radius of a spherical-symmetric mass distribution reads e.g., [55] Chap. XI, §97:

$$
R_{\mathrm{g}}=\frac{2 G M}{c^{2}}
$$

and related mean density is:

$$
\bar{\rho}_{\mathrm{g}}=\frac{3 M}{4 \pi R_{\mathrm{g}}^{3}}=\frac{3}{32 \pi} \frac{c^{6}}{G^{3} M^{2}} ;
$$


which scales as an inverse square mass.

Using Eq. (9), the mass as a function of the gravitational radius reads:

$$
\frac{M}{m_{\odot}}=\frac{c^{2}}{2 G m_{\odot}} \frac{R_{\mathrm{g}}}{\mathrm{m}}=\frac{R_{\mathrm{g}} / \mathrm{m}}{\left(R_{\mathrm{g}}\right)_{\odot} / \mathrm{m}}=\frac{R_{\mathrm{g}} / \mathrm{km}}{\left(R_{\mathrm{g}}\right)_{\odot} / \mathrm{km}}=0.338604 \frac{R_{\mathrm{g}}}{\mathrm{km}}
$$

which is plotted in Figs. 2 and 3 as a dashed line.

The gravitational radius, $R_{\mathrm{g}}$, via Eqs. (8) and (9) can be expressed in terms of related density parameter, $\left(\zeta_{\mathrm{n}}\right)_{\mathrm{g}}$, as:

$$
\frac{R_{\mathrm{g}}}{\mathrm{km}}=\left[\frac{c^{2}}{2 G m_{\mathrm{n}}}\left(\zeta_{\mathrm{n}}\right)_{\mathrm{g}} r_{\mathrm{n}}^{3}\right]^{1 / 2} \frac{1}{\mathrm{~km}}=20.0497\left[\left(\zeta_{\mathrm{n}}\right)_{\mathrm{g}}\right]^{1 / 2}
$$

and related mass reads:

$$
\frac{M}{m_{\odot}}=\frac{c^{2} R_{\mathrm{g}}}{2 G m_{\odot}}=\frac{c^{2}}{2 G m_{\odot}}\left[\frac{c^{2}\left(\zeta_{\mathrm{n}}\right)_{\mathrm{g}} r_{\mathrm{n}}^{3}}{2 G m_{\mathrm{n}}}\right]^{1 / 2}=6.7889\left[\left(\zeta_{\mathrm{n}}\right)_{\mathrm{g}}\right]^{1 / 2}
$$

accordingly, $\left(R_{\mathrm{g}} / \mathrm{km}, M / m_{\odot}\right)=\left\{20.0497\left[\left(\zeta_{\mathrm{n}}\right)_{\mathrm{g}}\right]^{1 / 2}, 6.7889\left[\left(\zeta_{\mathrm{n}}\right)_{\mathrm{g}}\right]^{1 / 2}\right\}$ are NS gravitational radius and mass for assigned density factor, $\left(\zeta_{\mathrm{n}}\right)_{\mathrm{g}}$.

Casual relationship may safely be expressed as e.g., [58] [91]:

$$
\frac{M}{m_{\odot}}=0.24 \frac{R}{\mathrm{~km}}
$$

which is plotted in Figs. 2 and 3 as a dotted line. Larger masses at fixed radius imply causality violation.

Let $M$ and $\bar{\rho}$ be mass and mean density, respectively, of a generic body. The radius, $R_{\mathrm{s}}$, and the free-fall time, $\left(t_{\mathrm{s}}\right)_{\mathrm{ff}}$, of a dust sphere of equal mass and mean density read e.g., [6]:

$$
\begin{aligned}
& R_{\mathrm{s}}=\left(\frac{3}{4 \pi} \frac{M}{\bar{\rho}}\right)^{1 / 3} ; \\
& \left(t_{\mathrm{s}}\right)_{\mathrm{ff}}=\left(\frac{3 \pi}{32} \frac{1}{G \bar{\rho}}\right)^{1 / 2} ;
\end{aligned}
$$

respectively.

The particularization to $\bar{\rho}=\bar{\rho}_{g}$ and the combination with Eqs. (9) and (10) yields after little algebra:

$$
\left(t_{\mathrm{g}}\right)_{\mathrm{ff}}=\left(\frac{3 \pi}{32} \frac{1}{G \bar{\rho}_{\mathrm{g}}}\right)^{1 / 2}=\frac{\pi}{2} \frac{R_{\mathrm{g}}}{c}
$$


which reads $\left(t_{\mathrm{g}}\right)_{\mathrm{ff}} \approx 0.015 \mathrm{~ms}$ for the sun.

The combination of Eqs. (9), (15), (16), after some algebra yields:

$$
\frac{R_{\mathrm{s}}}{R_{\mathrm{g}}}=\left(\frac{M_{0}^{2}}{M^{2}} \frac{\bar{\rho}_{0}}{\bar{\rho}}\right)^{1 / 3} ; \quad M_{0}^{2} \bar{\rho}_{0}=\frac{3}{32 \pi} \frac{c^{6}}{G^{3}} ;
$$

which scales as $M^{-2 / 3} \bar{\rho}^{-1 / 3}$.

For fixed $\bar{\rho}$, Eq. (18) reduces to:

$$
\frac{R_{\mathrm{s}}}{R_{\mathrm{g}}}=\left(\frac{M_{0}}{M}\right)^{2 / 3} ; \quad M_{0}=\frac{c^{3}\left(t_{\mathrm{s}}\right)_{\mathrm{ff}}}{\pi G} ;
$$

which scales as $M^{-2 / 3}$.

For fixed $M$, Eq. (18) reduces to:

$$
\frac{R_{\mathrm{s}}}{R_{\mathrm{g}}}=\left(\frac{\bar{\rho}_{0}}{\bar{\rho}}\right)^{1 / 3} \quad ; \quad \bar{\rho}_{0}=\frac{3}{32 \pi} \frac{c^{6}}{G^{3} M^{2}} ;
$$

which scales as $\bar{\rho}^{-1 / 3}$.

Turning to the general case, the threshold of inescapable gravitational collapse is $R_{\mathrm{s}}=R_{\mathrm{g}}$ and Eq. (18) reduces to $M^{2} \bar{\rho}=M_{0}^{2} \bar{\rho}_{0}$ or:

$$
\log \frac{\bar{\rho}}{\mathrm{kg} / \mathrm{m}^{3}}=\log \left(\frac{3}{32 \pi} \frac{c^{6}}{G^{3}} \frac{\mathrm{m}^{3}}{\mathrm{~kg}^{3}}\right)-2 \log \frac{M}{\mathrm{~kg}} ;
$$

which is shown in Fig. 4 as a full line. Dotted horizontal lines mark neutron mean density, $\bar{\rho}_{\mathrm{n}}=3.9988 \cdot 10^{17} \mathrm{~kg} / \mathrm{m}^{3}$, and water mean density, $\bar{\rho}_{\mathrm{w}}=0.9998$. $10^{3} \mathrm{~kg} / \mathrm{m}^{3}$, respectively.

For comparison, the following bodies are also plotted in Fig.4 as diamonds: neutron (n); hail bead (hb); comet (67P/Churyumov-Gerasimenko, 67P/CG); Moon (M); Earth (E); Jupiter (J); Sun (S); white dwarf (Syrius B, $\mathrm{SB}$ ); neutron star (PSR J0337+1715, assumed radius, NS); globular cluster (M13, M13); galaxy (Milky Way, MW); galaxy cluster (Coma, C). Related data are listed in Table 1 .

An inspection of Fig. 4 discloses NSs (including QSs and hybrid stars) are systems (where laws of ordinary physics hold) closest to BHs (where laws of ordinary physics no longer hold).

\section{About black holes}

$\mathrm{BH}$ progenitors are characterized by inescapable gravitational collapse where, from the standpoint of general relativity, the final configuration may be conceived as a mass point, or spindle, or flat disk. Related observables are mass, 
Table 1: Mass, $M$; mean density, $\bar{\rho}$; radius of associated sphere, $R_{\mathrm{s}}$; normalized radius of associated sphere with respect to gravitational radius of associated sphere, $R_{\mathrm{s}} / R_{\mathrm{g}}$; of selected bodies ranging from micro to macro cosmos. Symbol caption: n - neutron; hb - hail bean; 67P/CG - comet (67P/Churyumov-Gerasimenko); M - Moon; E - Earth; J - Jupiter; S - Sun; SB - white dwarf (Syrius B); NS - neutron star (PSR J0337+1715, assumed radius); M13 - globular cluster (M13); MW - galaxy (Milky Way); C - galaxy cluster (Coma).

\begin{tabular}{lcccc}
\hline symbol & $M / \mathrm{kg}$ & $\bar{\rho} /\left(\mathrm{kg} / \mathrm{m}^{3}\right)$ & $R_{\mathrm{s}} / \mathrm{m}$ & $R_{\mathrm{s}} / R_{\mathrm{g}}$ \\
\hline $\mathrm{n}$ & $1.6749 \mathrm{E}-27$ & $3.9988 \mathrm{E}+17$ & $1.0000 \mathrm{E}-15$ & $4.8729 \mathrm{E}+60$ \\
$\mathrm{hb}$ & $1.0000 \mathrm{E}-02$ & $9.1700 \mathrm{E}+02$ & $1.3757 \mathrm{E}-02$ & $1.1228 \mathrm{E}+49$ \\
$67 \mathrm{P} / \mathrm{CG}$ & $1.0000 \mathrm{E}+13$ & $4.7000 \mathrm{E}+02$ & $1.7190 \mathrm{E}+03$ & $1.1574 \mathrm{E}+17$ \\
$\mathrm{M}$ & $7.3420 \mathrm{E}+22$ & $3.3462 \mathrm{E}+03$ & $1.7367 \mathrm{E}+06$ & $1.5927 \mathrm{E}+10$ \\
$\mathrm{E}$ & $5.9726 \mathrm{E}+24$ & $5.5140 \mathrm{E}+03$ & $6.3709 \mathrm{E}+06$ & $7.1824 \mathrm{E}+08$ \\
$\mathrm{~J}$ & $1.8986 \mathrm{E}+27$ & $1.3260 \mathrm{E}+03$ & $6.9920 \mathrm{E}+07$ & $2.4797 \mathrm{E}+07$ \\
S & $1.9886 \mathrm{E}+30$ & $1.4112 \mathrm{E}+03$ & $6.9548 \mathrm{E}+08$ & $2.3549 \mathrm{E}+05$ \\
SB & $2.0243 \mathrm{E}+30$ & $2.4139 \mathrm{E}+09$ & $5.8500 \mathrm{E}+06$ & $1.9458 \mathrm{E}+03$ \\
NS & $2.8591 \mathrm{E}+30$ & $5.8963 \mathrm{E}+17$ & $1.0500 \mathrm{E}+04$ & $2.4728 \mathrm{E}+00$ \\
M13 & $1.1931 \mathrm{E}+36$ & $5.8469 \mathrm{E}-19$ & $7.8685 \mathrm{E}+17$ & $4.4405 \mathrm{E}+08$ \\
$\mathrm{MW}$ & $1.3562 \mathrm{E}+42$ & $2.5446 \mathrm{E}-21$ & $5.0297 \mathrm{E}+20$ & $2.4972 \mathrm{E}+05$ \\
$\mathrm{C}$ & $4.3549 \mathrm{E}+45$ & $5.5292 \mathrm{E}-25$ & $1.2343 \mathrm{E}+23$ & $1.9083 \mathrm{E}+04$ \\
\hline
\end{tabular}


angular momentum, and electric charge, leaving aside thermodynamics. The situation is far more complex in the framework of superstring theories, where 7 additional dimensions are taken into consideration, and supersymmetric (SUSY) theories, where gravitational, electromagnetic, weak and strong interaction can be unified. For further details, an interested reader is addressed to specific textbooks e.g., [45]. In any case, BHs may safely be thought of as single entities, according to the following considerations.

Particles lose their identity within BHs, in the sense their self energy is negligible with respect to tidal energy from BH. Matter therein fills so tiny volume that extra dimensions (if any) are comparable to usual dimensions, implying BHs attain a pre-big bang state. Then the four known interactions therein are expected to be unified into a single interaction i.e. supergravity. Outside event horizon, supergravity necessarily reduces to ordinary gravity and the four usual dimensions dominate on the hidden remaining seven.

Rotating BHs in Kerr metric are characterized by spin parameter e.g., [110 87 as:

$$
a_{\mathrm{BH}}^{*}=\frac{c J_{\mathrm{BH}}}{G M_{\mathrm{BH}}^{2}}=\frac{c^{2}}{2 G M_{\mathrm{BH}}} \frac{2 J_{\mathrm{BH}}}{c M_{\mathrm{BH}}}=\frac{2 J_{\mathrm{BH}}}{c M_{\mathrm{BH}} R_{\mathrm{g}}} \quad ; \quad 0 \leq a_{\mathrm{BH}}^{*} \leq 1 ;
$$

accordingly, $\mathrm{BH}$ angular momentum reads:

$$
J_{\mathrm{BH}}=\frac{1}{2} M_{\mathrm{BH}} a_{\mathrm{BH}}^{*} c R_{\mathrm{g}} .
$$

In classical mechanics, the angular momentum of a rigidly rotating (in particular, infinitely thin) homogeneous disk is:

$$
J_{\mathrm{d}}=\frac{1}{2} M_{\mathrm{d}} v_{\mathrm{d}} R_{\mathrm{d}}
$$

where $v_{\mathrm{d}}=\Omega_{\mathrm{d}} R_{\mathrm{d}}$ is disk equatorial velocity and $T_{\mathrm{d}}=2 \pi / \Omega_{\mathrm{d}}=2 \pi R_{\mathrm{d}} / v_{\mathrm{d}}$ is disk period.

A principle of corresponding states can be established via Eqs. (23) and (24) between spinning BHs and rigidly rotating, infinitely thin, homogeneous disks.

Spinning BHs in Kerr metrics can be related to rigidly rotating, infinitely thin, homogeneous disks in classical mechanics, of equal mass, $M_{\mathrm{d}}=M_{\mathrm{BH}}$, radius equal to gravitational radius, $R_{\mathrm{d}}=R_{\mathrm{g}}$, and equatorial velocity, $v_{\mathrm{d}}=$ $a_{\mathrm{BH}}^{*} c, 0 \leq a_{\mathrm{BH}}^{*} \leq 1$. 
In this view, $\mathrm{BH}$ period and moment of inertia read:

$$
\begin{aligned}
& T_{\mathrm{BH}}=\frac{2 \pi}{\Omega_{\mathrm{BH}}}=\frac{2 \pi}{a_{\mathrm{BH}}^{*} c / R_{\mathrm{g}}}=\frac{2 \pi}{a_{\mathrm{BH}}^{*} c} \frac{2 G M_{\mathrm{BH}}}{c^{2}}=\frac{4 \pi G M_{\mathrm{BH}}}{a_{\mathrm{BH}}^{*} c^{3}} ; \\
& I_{\mathrm{BH}}=\frac{1}{2} M_{\mathrm{BH}} R_{\mathrm{g}}^{2}=\frac{1}{2} M_{\mathrm{BH}}\left(\frac{2 G M_{\mathrm{BH}}}{c^{2}}\right)^{2}=\frac{2 G^{2} M_{\mathrm{BH}}^{3}}{c^{4}} ;
\end{aligned}
$$

which allow comparison with NS/QS counterparts.

To this respect, it is worth remembering descriptions in general relativity can be performed, in some special cases, using classical concepts. For instance, a logarithmic gravitational potential, together with an allowance for space-time curvature, provide laws of motion for a free particle near a nonrotating $\mathrm{BH}$, which coincide with their counterparts formulated in general relativity [101.

With regard to stable maximum mass configurations of fixed spin frequency, calculations were performed in a recent investigation [63] for axisymmetric, rigidly rotating NSs and QSs, using 4 and 3 different EOSs, respectively.

Let $M_{\max }$ be maximum mass of equilibrium configurations mentioned above, and let $R_{\max }, I_{\max }$, be related equatorial radius and moment of inertia, for assigned angular velocity, $\Omega$. Let $M_{\mathrm{TOV}}, R_{\mathrm{TOV}}, I_{\mathrm{TOV}}$, be their counterparts in the special case of zero spin frequency i.e. nonrotating configurations.

The dependence of $\left(M_{\max }, R_{\max }, I_{\max }\right)$ on angular velocity or rotation period can be approximated by the following relations [63]:

$$
\begin{aligned}
& \frac{M_{\max }}{m_{\odot}}=\frac{M_{\mathrm{TOV}}}{m_{\odot}}\left[1+\alpha\left(\frac{T}{\mathrm{~ms}}\right)^{\beta}\right] ; \\
& \frac{R_{\max }}{\mathrm{km}}=C+A\left(\frac{T}{\mathrm{~ms}}\right)^{B} ; \\
& \frac{I_{\max }}{10^{38} \mathrm{~kg} \mathrm{~m}^{2}}=\frac{M_{\max }}{m_{\odot}}\left(\frac{R_{\max }}{\mathrm{km}}\right)^{2} \frac{a C_{\odot} / 100}{1+\exp [-k T(1-q / T)]} ;
\end{aligned}
$$

where $T=2 \pi / \Omega$ is period, $C_{\odot}$ is solar mass coefficient, and remaining parameters depend on EOS. Related values are listed in the parent paper [63] Table 1 and an interested reader is addressed therein for further details.

The following profile parameter:

$$
\eta=\frac{I}{M R_{\mathrm{eq}}^{2}}=\frac{I \Omega}{M R_{\mathrm{eq}}^{2} \Omega}=\frac{J}{M R_{\mathrm{eq}}^{2} v_{\mathrm{eq}} / R_{\mathrm{eq}}}=\frac{J}{M v_{\mathrm{eq}} R_{\mathrm{eq}}} ;
$$


where $R_{\text {eq }}$ is equatorial radius and $J$ angular momentum assuming rigid rotation, via Eq. (29) reads in particular:

$$
\begin{aligned}
\eta_{\max } & =\frac{I_{\max }}{M_{\max } R_{\max }^{2}}=\frac{I_{\max } /\left(10^{38} \mathrm{~kg} \mathrm{~m}^{2}\right) 10^{38} \mathrm{~kg} \mathrm{m^{2 }}}{\left(M_{\max } / m_{\odot}\right) m_{\odot}\left(R_{\max } / \mathrm{km}^{2} \mathrm{~km}^{2}\right.} \\
& =\frac{a C_{\odot} / 100}{1+\exp \left[-k T_{\max }\left(1-q / T_{\max }\right)\right]} \frac{10^{38} \mathrm{~kg} \mathrm{~m}^{2}}{C_{\odot} 10^{30} \mathrm{~kg} 10^{6} \mathrm{~m}^{2}} \\
& =\frac{a}{1+\exp \left[-k T_{\max }\left(1-q / T_{\max }\right)\right]}
\end{aligned}
$$

with regard to maximum mass configurations for assigned angular velocity.

The special case of TOV and equatorial breakup (EQB) configuration reads:

$$
\begin{aligned}
& \eta_{\mathrm{TOV}}=\frac{I_{\mathrm{TOV}}}{M_{\mathrm{TOV}} R_{\mathrm{TOV}}^{2}}=a ; \\
& \eta_{\mathrm{EQB}}=\frac{I_{\mathrm{EQB}}}{M_{\mathrm{EQB}} R_{\mathrm{EQB}}^{2}}=\frac{a}{1+\exp \left[-k T_{\mathrm{EQB}}\left(1-q / T_{\mathrm{EQB}}\right)\right]} ;
\end{aligned}
$$

respectively, where $T_{\mathrm{EQB}}$ is the spin period at equatorial breakup.

Realistic values of $I_{\mathrm{TOV}}$ and $\eta_{\mathrm{TOV}}$ imply the parameter, $a$, has to be divided by $100 / C_{\odot}$ according to Eq. (29). In fact, related values listed in the parent paper 63] Table 1 have to be read as $\eta_{\text {TOV }}$ instead of $a$ according to Eq. (32).

The counterpart of $\mathrm{BH}$ spin parameter, $a_{\mathrm{BH}}^{*}$, defined by Eq. (22) , for NS/QS reads:

$$
a_{\mathrm{NQ}}^{*}=\frac{c J}{G M^{2}}
$$

which, in the case under discussion of rigid rotation, reduces to:

$$
a_{\mathrm{NQ}}^{*}=\frac{c I \Omega}{G M^{2}}=\frac{2 \pi c I}{G M^{2} T} .
$$

In convenient units, Eq. (35) translates into:

$$
\begin{aligned}
a_{\mathrm{NQ}}^{*} & =\frac{2 \pi C_{\mathrm{c}}\left(10^{8} \mathrm{~m} / \mathrm{s}\right) I /\left(10^{38} \mathrm{~kg} \mathrm{~m}^{2}\right) 10^{38} \mathrm{~kg} \mathrm{~m}^{2}}{C_{\mathrm{G}}\left[10^{-11} \mathrm{~m}^{3} /\left(\mathrm{kg} \mathrm{s}^{2}\right)\right]\left(M / m_{\odot}\right)^{2}\left(C_{\odot} 10^{30} \mathrm{~kg}\right)^{2}(T / \mathrm{ms}) 10^{-3} \mathrm{~s}} \\
& =\frac{2 \pi C_{\mathrm{c}}}{C_{\mathrm{G}} C_{\odot}^{2}} \frac{I /\left(10^{38} \mathrm{~kg} \mathrm{~m}^{2}\right)}{\left(M / m_{\odot}\right)^{2}(T / \mathrm{ms})} ; \\
c= & C_{\mathrm{c}} 10^{8} \mathrm{~m} / \mathrm{s} ; \quad G=C_{\mathrm{G}} 10^{-11} \mathrm{~m}^{3} /\left(\mathrm{kg} \mathrm{s}^{2}\right) ; \quad m_{\odot}=C_{\odot} 10^{30} \mathrm{~kg} ;
\end{aligned}
$$

where $2 \pi C_{\mathrm{c}} /\left(C_{\mathrm{G}} C_{\odot}^{2}\right)=0.713893$. 
Table 2: Comparison between TOV and EQB configuration parameters (first and second line of each panel) and additional parameters of EQB configurations (third line of each panel). Parameters are specified on the head of the Table as: mass, equatorial radius, moment of inertia, profile parameter (first and second line of each panel), and: angular momentum, specific angular momentum, spin parameter, period (third line of each panel). EOSs related to each panel are (from top to bottom): BCPM, BSk20, BSk21, Shen, CIDDM, CDDM1, CDDM2, where the first four are applied to NSs and the last three to QSs. Data are taken or inferred from the parent paper [63]. See text for further details.

\begin{tabular}{|c|c|c|c|}
\hline $\begin{array}{c}M_{\mathrm{TOV}} / m_{\odot} \\
M_{\mathrm{EQB}} / m_{\odot} \\
J_{\mathrm{EQB}} /\left(10^{42} \mathrm{~kg} \mathrm{~m}^{2} / \mathrm{s}\right) \\
\end{array}$ & $\begin{array}{c}R_{\mathrm{TOV}} / \mathrm{km} \\
R_{\mathrm{EQB}} / \mathrm{km} \\
j_{\mathrm{EQB}} /\left(10^{12} \mathrm{~m}^{2} / \mathrm{s}\right) \\
\end{array}$ & $\begin{array}{c}I_{\mathrm{TOV}} /\left(10^{38} \mathrm{~kg} \mathrm{~m}^{2}\right) \\
I_{\mathrm{EQB}} /\left(10^{38} \mathrm{~kg} \mathrm{~m}^{2}\right) \\
a_{\mathrm{EQB}}^{*} \\
\end{array}$ & $\begin{array}{c}\eta_{\mathrm{TOV}} \\
\eta_{\mathrm{EQB}} \\
T_{\mathrm{EQB}} / \mathrm{ms}\end{array}$ \\
\hline $1.98000 \mathrm{D}+00$ & $9.94100 \mathrm{D}+00$ & $1.75445 \mathrm{D}+00$ & $4.50900 \mathrm{D}-01$ \\
\hline $2.33809 \mathrm{D}+00$ & $1.33165 \mathrm{D}+01$ & $2.85700 \mathrm{D}+00$ & $3.46524 \mathrm{D}-01$ \\
\hline $1.37494 \mathrm{D}+00$ & $6.91429 \mathrm{D}-01$ & $6.68154 \mathrm{D}-01$ & $5.58400 \mathrm{D}-01$ \\
\hline $2.17000 \mathrm{D}+00$ & $1.01700 \mathrm{D}+01$ & $2.10391 \mathrm{D}+00$ & $4.71400 \mathrm{D}-01$ \\
\hline $2.57642 \mathrm{D}+00$ & $1.34191 \mathrm{D}+01$ & $3.50300 \mathrm{D}+00$ & $3.79699 \mathrm{D}-01$ \\
\hline $1.58465 \mathrm{D}+00$ & $7.96888 \mathrm{D}-01$ & $6.98828 \mathrm{D}-01$ & $5.39100 \mathrm{D}-01$ \\
\hline $2.28000 \mathrm{D}+00$ & $1.10800 \mathrm{D}+01$ & $2.69288 \mathrm{D}+00$ & $4.83800 \mathrm{D}-01$ \\
\hline $2.72701 \mathrm{D}+00$ & $1.47324 \mathrm{D}+01$ & $4.36800 \mathrm{D}+00$ & $3.71120 \mathrm{D}-01$ \\
\hline $1.67150 \mathrm{D}+00$ & $8.40564 \mathrm{D}-01$ & $6.96425 \mathrm{D}-01$ & $6.02100 \mathrm{D}-01$ \\
\hline $2.18000 \mathrm{D}+00$ & $1.24000 \mathrm{D}+01$ & $2.73421 \mathrm{D}+00$ & $4.10200 \mathrm{D}-01$ \\
\hline $2.59936 \mathrm{D}+00$ & $1.68886 \mathrm{D}+01$ & $4.67500 \mathrm{D}+00$ & $3.17095 \mathrm{D}-01$ \\
\hline $1.58203 \mathrm{D}+00$ & $7.95568 \mathrm{D}-01$ & $6.91513 \mathrm{D}-01$ & $7.14300 \mathrm{D}-01$ \\
\hline $2.09000 \mathrm{D}+00$ & $1.24300 \mathrm{D}+01$ & $2.84658 \mathrm{D}+00$ & $4.43300 \mathrm{D}-01$ \\
\hline $2.92295 \mathrm{D}+00$ & $1.94749 \mathrm{D}+01$ & $8.64500 \mathrm{D}+00$ & $3.92154 \mathrm{D}-01$ \\
\hline $2.23196 \mathrm{D}+00$ & $1.12241 \mathrm{D}+00$ & 8.67599D-01 & $8.32600 \mathrm{D}-01$ \\
\hline $2.21000 \mathrm{D}+00$ & $1.39900 \mathrm{D}+01$ & $3.65813 \mathrm{D}+00$ & $4.25300 \mathrm{D}-01$ \\
\hline $3.09282 \mathrm{D}+00$ & $2.24108 \mathrm{D}+01$ & $1.16700 \mathrm{D}+01$ & $3.77805 \mathrm{D}-01$ \\
\hline $2.38033 \mathrm{D}+00$ & $1.19702 \mathrm{D}+00$ & $8.74453 \mathrm{D}-01$ & $9.96000 \mathrm{D}-01$ \\
\hline $2.45000 \mathrm{D}+00$ & $1.57600 \mathrm{D}+01$ & $5.08840 \mathrm{D}+00$ & $4.20500 \mathrm{D}-01$ \\
\hline $3.44237 \mathrm{D}+00$ & $2.51923 \mathrm{D}+01$ & $1.63400 \mathrm{D}+01$ & $3.76116 \mathrm{D}-01$ \\
\hline $2.65131 \mathrm{D}+00$ & $1.33329 \mathrm{D}+00$ & $8.75097 \mathrm{D}-01$ & $1.12490 \mathrm{D}+00$ \\
\hline
\end{tabular}


Table 3: Mass, $M$, radius, $R$, gravitational radius, $R_{\mathrm{g}}$, moment of inertia, $I$, compactness parameter, $\beta^{*}=(1 / 2)\left(R_{\mathrm{g}} / R\right)$, profile parameter, $\eta=I /\left(M R^{2}\right)$, inferred for PSR J0737-3039A [92] (first row) and PSR J03478+0432 [122] for lower and upper mass limit (second and third row, respectively). See text for further details.

\begin{tabular}{llllcc}
\hline$M / m_{\odot}$ & $R / \mathrm{km}$ & $R_{\mathrm{g}} / \mathrm{km}$ & $I /\left(10^{38} \mathrm{~kg} \mathrm{~m}^{2}\right)$ & $\beta^{*}$ & $\eta$ \\
\hline 1.338 & 13 & 3.951 & 1.5 & 0.1520 & 0.3336 \\
1.97 & 12.957 & 5.817 & 1.906 & 0.2245 & 0.2898 \\
2.05 & 12.143 & 6.053 & 1.557 & 0.2492 & 0.2557 \\
\hline
\end{tabular}

Parameters related to TOV and EQB configurations, for seven different EOSs, are listed in Table 2, where data are taken or inferred from the parent paper [63]. More specifically, different panels correspond to different EOSs concerning NSs (first four) and QSs (last three). Masses, equatorial radii, moments of inertia, profile parameters, for TOV and EQB configurations, are listed on the first and second line of each panel. Angular momenta, specific angular momenta, spin parameters, periods, for EQB configurations, are listed on the third line of each panel. As outlined in the parent paper [63, the effect of rotation is increasing mass, equatorial radius, and moment of inertia. On the other hand, the profile parameter, $\eta$, appears to be lowered by rotation.

The profile parameter, $\eta$, can empirically be related to the compactness parameter, $\beta^{*}=(1 / 2)\left(R_{\mathrm{g}} / R\right)$, as:

$$
\eta=(0.237 \mp 0.008)\left[1+2.844 \beta^{*}+18.910\left(\beta^{*}\right)^{4}\right] ;
$$

for a large EOS set [57] [104] [105], and:

$$
\eta=0.207+0.857 \beta^{*} \mp 0.011
$$

for a limited set of microscopic EOS [114].

Values of $\left(\beta^{*}, \eta\right)$ inferred for single objects are shown in Table 3, where the first row relates to PSR J0737-3039A [92] and the other two to PSR J03478+0432 [122] for lower and upper mass limit, respectively. In the former case, a fiducial value of moment of inertia has been assumed together with a consistent fiducial value of radius. For further details, an interested reader is addressed to the parent paper [92].

The compactness parameter, $\beta^{*}$, can be inferred from results listed in Table 2: related $\left(\beta^{*}, \eta\right)$ are plotted in Fig. 5 as diamonds (TOV) and squares 
(EQB). Empirical fits via Eqs. (38) and (39) are shown as a dashed and full band, respectively. Also included for comparison are PSR J0737-3039A (asterisk) and PSR J0348+0432 (triangles) related to lower (left) and upper (right) mass limit.

An inspection of Fig. 5 discloses TOV configurations are consistent or marginally inconsistent with the above mentioned fits, while EQB configurations are consistent for QSs and largely inconsistent (but lying on a parallel sequence) for NSs. It is worth remembering classical and general relativistic limit relate to $\beta^{*} \rightarrow 0$ and $\beta^{*} \rightarrow 1 / 2$, respectively. Dotted lines parallel to the full band are shown for comparison with lower EQB configurations (intercept arbitrarily chosen equal to 0.1343) and for fitting BH limit, $\left(\beta^{*}, \eta\right)=(1 / 2,1 / 2)$ (intercept equal to 0.0715$)$.

With regard to BHs in Kerr metric, the particularization of Eqs. (23), (25), (26) , (30), to nonrotating $\left(a_{\mathrm{BH}}^{*}=0\right)$ and maximally rotating $\left(a_{\mathrm{BH}}^{*}=1\right)$ $\mathrm{BHs}$ yields:

$$
\begin{aligned}
J_{\mathrm{EQB}} & =\frac{1}{2} M_{\mathrm{EQB}} c\left(R_{\mathrm{g}}\right)_{\mathrm{EQB}} ; \\
T_{\mathrm{EQB}} & =\frac{2 \pi\left(R_{\mathrm{g}}\right)_{\mathrm{EQB}}}{c} ; \\
I_{\mathrm{TOV}} & =\frac{1}{2} M_{\mathrm{TOV}}\left[\left(R_{\mathrm{g}}\right)_{\mathrm{TOV}}\right]^{2} ; \\
I_{\mathrm{EQB}} & =\frac{1}{2} M_{\mathrm{EQB}}\left[\left(R_{\mathrm{g}}\right)_{\mathrm{EQB}}\right]^{2} ; \\
\eta_{\mathrm{TOV}} & =\eta_{\mathrm{EQB}}=\frac{1}{2} ;
\end{aligned}
$$

where, aiming to preserve continuity, TOV configurations are conceived as nonrotating disks of the kind considered instead of nonrotating homogeneous spheres implying $I_{\mathrm{TOV}}=(2 / 5) M_{\mathrm{TOV}}\left[\left(R_{\mathrm{g}}\right)_{\mathrm{TOV}}\right]^{2}$.

Parameters related to TOV and EQB configurations (in the above specified sense), for masses equal to their NS/QS counterparts listed in Table 2, are presented in Table 4. Masses, gravitational radii, moments of inertia, profile parameters, for TOV and EQB configurations are listed on the first and second line in each panel. Angular momenta, specific angular momenta, spin parameters, periods, for EQB configurations are listed on the third line of each panel.

For maximally rotating $\left(a_{\mathrm{BH}}^{*}=1\right) \mathrm{BHs}$, Eq. (23) via (9) and (37) reduces to:

$$
\frac{J_{\mathrm{BH}}}{10^{42} \mathrm{~kg} \mathrm{~m}^{2} / \mathrm{s}} 10^{42} \frac{\mathrm{kg} \mathrm{m}^{2}}{\mathrm{~s}}=\frac{G\left(M / m_{\odot}\right)^{2} m_{\odot}^{2}}{c}
$$


Table 4: Comparison between TOV and EQB configuration parameters (first and second line of each panel) and additional parameters of EQB configurations (third line of each panel), for BHs of equal mass with respect to their NS/QS counterparts. Parameters are specified on the head of the Table as: mass, equatorial radius, moment of inertia, profile parameter (first and second line of each panel), and: angular momentum, specific angular momentum, spin parameter, period (third line of each panel). EOSs related to each panel are (from top to bottom): BCPM, BSk20, BSk21, Shen, CIDDM, CDDM1, CDDM2, where the first four are applied to NSs and the last three to QSs. Data are taken or inferred from the parent paper [63]. See text for further details.

\begin{tabular}{lccc}
\hline$M_{\mathrm{TOV}} / m_{\odot}$ & $\left(R_{\mathrm{g}}\right)_{\mathrm{TOV}} / \mathrm{km}$ & $I_{\mathrm{TOV}} /\left(10^{38} \mathrm{~kg} \mathrm{~m}^{2}\right)$ & $\eta_{\mathrm{TOV}}$ \\
$M_{\mathrm{EQB}} / m_{\odot}$ & $\left(R_{\mathrm{g}}\right)_{\mathrm{EQB}} / \mathrm{km}^{2}$ & $I_{\mathrm{EQB}} /\left(10^{38} \mathrm{~kg} \mathrm{~m}^{2}\right)$ & $\begin{array}{c}\eta_{\mathrm{EQB}} \\
T_{\mathrm{EQB}} / \mathrm{ms}\end{array}$ \\
$J_{\mathrm{EQB}} /\left(10^{42} \mathrm{~kg} \mathrm{~m}^{2} / \mathrm{s}\right)$ & $j_{\mathrm{EQB}} /\left(10^{12} \mathrm{~m}^{2} / \mathrm{s}\right)$ & $a_{\mathrm{EQB}}^{*}$ & $5.00000 \mathrm{D}-01$ \\
$1.98000 \mathrm{D}+00$ & $5.84635 \mathrm{D}+00$ & $6.72886 \mathrm{D}-01$ & $5.00000 \mathrm{D}-01$ \\
$2.33809 \mathrm{D}+00$ & $6.90367 \mathrm{D}+00$ & $1.10797 \mathrm{D}+00$ & $1.44690 \mathrm{D}-01$ \\
$4.81136 \mathrm{D}+00$ & $1.03483 \mathrm{D}+00$ & $1.00000 \mathrm{D}+00$ & $5.00000 \mathrm{D}-01$ \\
\hline $2.17000 \mathrm{D}+00$ & $6.40736 \mathrm{D}+00$ & $8.85778 \mathrm{D}-01$ & $5.00000 \mathrm{D}-01$ \\
$2.57642 \mathrm{D}+00$ & $7.60740 \mathrm{D}+00$ & $1.48250 \mathrm{D}+00$ & $1.59439 \mathrm{D}-01$ \\
$5.84225 \mathrm{D}+00$ & $1.14032 \mathrm{D}+00$ & $1.00000 \mathrm{D}+00$ & $5.00000 \mathrm{D}-01$ \\
\hline $2.28000 \mathrm{D}+00$ & $6.73216 \mathrm{D}+00$ & $1.02743 \mathrm{D}+00$ & $5.00000 \mathrm{D}-01$ \\
$2.72701 \mathrm{D}+00$ & $8.05204 \mathrm{D}+00$ & $1.75794 \mathrm{D}+00$ & $1.68758 \mathrm{D}-01$ \\
$6.54515 \mathrm{D}+00$ & $1.20697 \mathrm{D}+00$ & $1.00000 \mathrm{D}+00$ & $5.00000 \mathrm{D}-01$ \\
$2.18000 \mathrm{D}+00$ & $6.43689 \mathrm{D}+00$ & $8.98080 \mathrm{D}-01$ & $5.00000 \mathrm{D}-01$ \\
$2.59936 \mathrm{D}+00$ & $7.67514 \mathrm{D}+00$ & $1.52246 \mathrm{D}+00$ & $1.60859 \mathrm{D}-01$ \\
$5.94676 \mathrm{D}+00$ & $1.15047 \mathrm{D}+00$ & $1.00000 \mathrm{D}+00$ & $5.00000 \mathrm{D}-01$ \\
$2.09000 \mathrm{D}+00$ & $6.17115 \mathrm{D}+00$ & $7.91379 \mathrm{D}-01$ & $5.00000 \mathrm{D}-01$ \\
$2.92295 \mathrm{D}+00$ & $8.63060 \mathrm{D}+00$ & $2.16476 \mathrm{D}+00$ & $1.80884 \mathrm{D}-01$ \\
$7.51951 \mathrm{D}+00$ & $1.29369 \mathrm{D}+00$ & $1.00000 \mathrm{D}+00$ & $1.0000 \mathrm{D}-01$ \\
\hline $2.21000 \mathrm{D}+00$ & $6.52547 \mathrm{D}+00$ & $9.35669 \mathrm{D}-01$ & 5.00000 \\
$3.09282 \mathrm{D}+00$ & $9.13216 \mathrm{D}+00$ & $2.56453 \mathrm{D}+00$ & $5.00000 \mathrm{D}-01$ \\
$8.41889 \mathrm{D}+00$ & $1.36888 \mathrm{D}+00$ & $1.00000 \mathrm{D}+00$ & $1.91396 \mathrm{D}-01$ \\
\hline $2.45000 \mathrm{D}+00$ & $7.23412 \mathrm{D}+00$ & $1.27481 \mathrm{D}+00$ & $5.00000 \mathrm{D}-01$ \\
$3.44237 \mathrm{D}+00$ & $1.01643 \mathrm{D}+01$ & $3.53605 \mathrm{D}+00$ & $5.00000 \mathrm{D}-01$ \\
$1.04295 \mathrm{D}+01$ & $1.52359 \mathrm{D}+00$ & $1.00000 \mathrm{D}+00$ & $2.13028 \mathrm{D}-01$ \\
\hline
\end{tabular}




$$
=\frac{C_{\mathrm{G}} 10^{-11}\left(M / m_{\odot}\right)^{2} C_{\odot}^{2} 10^{60}}{C_{\mathrm{c}} 10^{8}} \frac{\mathrm{kg} \mathrm{m}^{2}}{\mathrm{~s}} ;
$$

which after little calculus reads:

$$
\frac{J_{\mathrm{BH}}}{10^{42} \mathrm{~kg} \mathrm{~m}^{2} / \mathrm{s}}=\frac{C_{\mathrm{G}} C_{\odot}^{2}}{10 C_{\mathrm{c}}}\left(\frac{M}{m_{\odot}}\right)^{2} ;
$$

where $C_{\mathrm{G}} C_{\odot}^{2} /\left(10 C_{\mathrm{c}}\right)=0.880130$.

Turning Eq. (45) into logarithms yields:

$$
\begin{aligned}
& \log \frac{J_{\mathrm{BH}}}{10^{42} \mathrm{~kg} \mathrm{~m}^{2} / \mathrm{s}}=2 \log \frac{M}{m_{\odot}}+\log C_{\mathrm{G}}+2 \log C_{\odot}-\log C_{\mathrm{c}}-1 ; \\
& \log C_{\mathrm{G}}+2 \log C_{\odot}-\log C_{\mathrm{c}}-1=-0.05545 ;
\end{aligned}
$$

which is a straight line of slope, 2 , and intercept, -0.05545 , on the logarithmic plane $\left\{\mathrm{O} \log \left(M / m_{\odot}\right) \log \left[J /\left(10^{42} \mathrm{~kg} \mathrm{~m}^{2} / \mathrm{s}\right)\right]\right\}$.

Let a NS/QS rigidly rotate with assigned frequency, $\Omega=2 \pi / T$, for fixed EOS. Related maximally rotating configuration is defined by $\left(M_{\max }, R_{\max }, I_{\max }\right)$ according to Eqs. (27)-(29) 63] and, owing to rigid rotation, angular momentum reads $J_{\max }=I_{\max } \Omega=I_{\max } 2 \pi / T$ or, using Eq. (29):

$$
\frac{J_{\max }}{10^{42} \mathrm{~kg} \mathrm{~m}^{2} / \mathrm{s}}=\frac{2 \pi / 10}{T / \mathrm{ms}} \frac{M_{\max }}{m_{\odot}}\left(\frac{R_{\max }}{\mathrm{km}}\right)^{2} \frac{C_{\odot}}{100} \frac{a}{1+\exp [-k T(1-q / T)]} ;
$$

where $J_{\max }=J_{\mathrm{TOV}}=0$ for $\Omega=0$ and $J_{\max }=J_{\mathrm{EQB}}$ for $\Omega=\Omega_{\mathrm{EQB}}, T=T_{\mathrm{EQB}}$.

Related curves on the logarithmic plane, $\left[\mathrm{O} \log \left(M / m_{\odot}\right) \log \left(J / 10^{42} \mathrm{~kg} \mathrm{~m}^{2} / \mathrm{s}\right)\right]$, are plotted in Fig. [6] where ending points i.e. EQB configurations are shown as diamonds. Maximally rotating $\left(a_{\mathrm{BH}}^{*}=1\right)$ BHs of equal mass, $M_{\mathrm{BH}}=M_{\mathrm{EQB}}$, are shown as asterisks, which are placed on the dotted straight line, related to Eq. (46)). A parallel line (arbitrarily chosen at $\log a_{\mathrm{BH}}^{*}=-0.5545$ or $a_{\mathrm{BH}}^{*}=0.2789$ ) is placed downwards to facilitate comparison with EQB configurations. Related EOSs are, from the left to the right with regard to ending points: (a) NSs - BCPM, BSk20, Shen, BSk21; (b) QSs - CIDDM, CDMM1, CDMM2.

Interestingly, EQB configurations are aligned to a good extent for both NSs and QSs, but slope is lower with respect to maximally rotating BHs. Accordingly, EQB configurations can be conceived as maximally rotating configurations, regardless of EOS. Related angular momentum is lower with respect to their maximally rotating $\mathrm{BH}$ counterparts by a factor of about 3.5851. Finally, it is worth of note equatorial velocity of EQB configurations, $v_{\mathrm{EQB}}=\Omega_{\mathrm{EQB}} R_{\mathrm{EQB}}=2 \pi R_{\mathrm{EQB}} / T_{\mathrm{EQB}}$, inferred from Table 2 and Eq. (9), equals about one half the light velocity, $v_{\mathrm{EQB}} \approx c / 2$. 


\section{Quark-level fusion in heavy baryons}

According to a recent investigation, quark-level fusion with release of energy can occur as well as nuclear fusion, involving heavy baryons [50], see also [69]. More specifically, (i) fusion between two heavy baryons $\left(\Lambda_{\mathrm{c}}^{+}\right)$produces a doubly charmed baryon $\left(\Xi_{\mathrm{cc}}^{++}\right)$and a neutron $(n), \Lambda_{\mathrm{c}}^{+} \Lambda_{\mathrm{c}}^{+} \rightarrow \Xi_{\mathrm{cc}}^{++} n$, with an energy release of $12 \mathrm{MeV}$; (ii) fusion between a heavy $\left(\Lambda_{\mathrm{c}}^{+}\right)$and a heavier $\left(\Lambda_{\mathrm{b}}^{0}\right)$ baryon produces a charmed-bottomed baryon $\left(\Xi_{\mathrm{cb}}^{+}\right)$and a neutron $(\mathrm{n})$, $\Lambda_{\mathrm{c}}^{+} \Lambda_{\mathrm{b}}^{0} \rightarrow \Xi_{\mathrm{cb}}^{+} n$, with an energy release of $50 \mathrm{MeV}$; (iii) fusion between two heavier baryons $\left(\Lambda_{\mathrm{b}}^{0}\right)$ produces a doubly bottomed baryon $\left(\Xi_{\mathrm{bb}}^{0}\right)$ and a neutron $(n), \Lambda_{\mathrm{b}}^{0} \Lambda_{\mathrm{b}}^{0} \rightarrow \Xi_{\mathrm{bb}}^{0} n$, with an energy release of $138 \mathrm{MeV}$.

In general, a reaction can be written as $\Lambda_{\mathrm{u}} \Lambda_{\mathrm{v}} \rightarrow \Xi_{\mathrm{uv}} N$, where uv $=$ ss, $\mathrm{cc}, \mathrm{bb}, \mathrm{cb}$, and $N$ is a nucleon. The reaction, $\Lambda_{\mathrm{s}} \Lambda_{\mathrm{s}} \rightarrow \Xi_{\mathrm{ss}} N$, is endothermic with an energy release of $-23 \mathrm{MeV}$. For further details, an interested reader is addressed to the parent paper [50].

As discussed therein, energy release is largely determined by binding energy between heavy quarks. Related interactions take place via an effective two-body potential, which implies binding energy is determined by their reduced mass, $\mu_{\text {red }}=m\left(\Lambda_{\mathrm{u}}\right) m\left(\Lambda_{\mathrm{v}}\right) /\left[m\left(\Lambda_{\mathrm{u}}\right)+m\left(\Lambda_{\mathrm{v}}\right)\right]$, where $m\left(\Lambda_{\mathrm{u}}\right)$ and $m\left(\Lambda_{\mathrm{v}}\right)$ are masses of individual quarks. It can be seen energy release, $\Delta E$, linearly depends on reduced mass, $\mu_{\text {red }}$, as [50]:

$$
\frac{\Delta E}{\mathrm{MeV}}=-44.95+0.0726 \mu_{\mathrm{red}} ;
$$

with regard to the above mentioned quark-level fusion reactions, $\Lambda_{\mathrm{u}} \Lambda_{\mathrm{v}} \rightarrow$ $\Xi_{\mathrm{uv}} N$.

Given a NS/QS, let the following restrictive assumptions hold: (1) a substantial mass fraction, $M_{\mathrm{uv}} / M_{\mathrm{NS} / \mathrm{QS}}$, is in form of heavy baryon reagents, $\Lambda_{\mathrm{u}}, \mathrm{u}=\mathrm{s}, \mathrm{c}, \mathrm{b}$; and (2) if $\Lambda_{\mathrm{u}} \Lambda_{\mathrm{v}} \rightarrow \Xi_{\mathrm{uv}} N$ reactions take place, the extension to the whole mass, $M_{\mathrm{uv}}$, is immediate. Accordingly, the number of reactions involving $\Lambda_{\mathrm{u}}, \Lambda_{\mathrm{v}}$, reads:

$$
N_{\mathrm{uv}}=\frac{M_{\mathrm{uv}}}{m\left(\Lambda_{\mathrm{u}}\right)+m\left(\Lambda_{\mathrm{v}}\right)}
$$

where $\mathrm{u}, \mathrm{v}=\mathrm{s}, \mathrm{c}, \mathrm{b}$. The total energy, $E_{\mathrm{uv}}$, released from $\Lambda_{\mathrm{u}} \Lambda_{\mathrm{v}} \rightarrow \Xi_{\mathrm{uv}} N$ reactions throughout the whole mass, $M_{\mathrm{uv}}$, is:

$$
E_{\mathrm{uv}}=N_{\mathrm{uv}} \Delta E_{\mathrm{uv}} ;
$$

where $\Delta E_{\mathrm{uv}}$ is energy released from a single reaction.

Related values are listed in Table [5: mass of heavy baryons, $m\left(\Lambda_{\mathrm{u}}\right)$, $m\left(\Xi_{\mathrm{uv}}\right)$, involved in reactions, $\Lambda_{\mathrm{u}} \Lambda_{\mathrm{v}} \rightarrow \Xi_{\mathrm{uv}} N$; energy release, $\Delta E_{\mathrm{uv}}$; number 
Table 5: Mass of heavy baryons, $m\left(\Lambda_{\mathrm{u}}\right), m\left(\Xi_{\mathrm{uv}}\right)$, involved in reactions, $\Lambda_{\mathrm{u}} \Lambda_{\mathrm{v}} \rightarrow \Xi_{\mathrm{uv}} N$, where $N$ is a nucleon; energy release, $\Delta E_{\mathrm{uv}}$; number of reactions per solar mass of reagents, $N_{\mathrm{uv}}=m_{\odot} /\left[m\left(\Lambda_{\mathrm{u}}\right)+m\left(\Lambda_{\mathrm{v}}\right)\right]$; energy release per solar mass of reagents, $E_{\mathrm{uv}}=N_{\mathrm{uv}} \Delta E_{\mathrm{uv}}$; for the following combinations of heavy quarks: $\mathrm{uv}=\mathrm{ss}, \mathrm{cc}, \mathrm{bb}, \mathrm{cb}$. In the last case, reagent masses can be read on related left columns. Values on the first three rows are taken from the parent paper [50] and converted from $\mathrm{MeV}$ to $\mathrm{kg}$ (mass) and $\mathrm{J}$ (energy), $1 \mathrm{MeV}=1.78266270 \cdot 10^{-30} \mathrm{~kg}=1.60217733 \cdot 10^{-13} \mathrm{~J}$. See text for further details.

\begin{tabular}{lcccc}
\hline $\begin{array}{c}\text { reaction: } \\
\text { parameter }\end{array}$ & $\mathrm{uv}=\mathrm{ss}$ & $\mathrm{uv}=\mathrm{cc}$ & $\mathrm{uv}=\mathrm{bb}$ & $\mathrm{uv}=\mathrm{cb}$ \\
\hline$m\left(\Lambda_{\mathrm{u}}\right) / \mathrm{kg}$ & $+1.9989 \mathrm{E}-27$ & $4.0761 \mathrm{E}-27$ & $1.0018 \mathrm{E}-26$ & (left values) \\
$m\left(\Xi_{\mathrm{uv}}\right) / \mathrm{kg}$ & $+2.3440 \mathrm{E}-27$ & $6.4557 \mathrm{E}-27$ & $1.8115 \mathrm{E}-26$ & $1.2331 \mathrm{E}-26$ \\
$\Delta E_{\mathrm{uv}} / \mathrm{J}$ & $-3.7010 \mathrm{E}-12$ & $1.9226 \mathrm{E}-12$ & $2.2110 \mathrm{E}-11$ & $8.0109 \mathrm{E}-12$ \\
$N_{\mathrm{uv}}$ & $+4.9991 \mathrm{E}+56$ & $2.4393 \mathrm{E}+56$ & $9.9249 \mathrm{E}+55$ & $1.4109 \mathrm{E}+56$ \\
$E_{\mathrm{uv}} / \mathrm{J}$ & $-1.8502 \mathrm{E}+45$ & $4.6898 \mathrm{E}+44$ & $2.1784 \mathrm{E}+45$ & $1.1307 \mathrm{E}+45$ \\
\hline
\end{tabular}

of reactions per solar mass of reagents, $N_{\text {uv }}$; energy release per solar mass of reagents, $E_{\mathrm{uv}}$; for the following combinations of heavy quarks: uv $=\mathrm{ss}$, cc, $\mathrm{bb}, \mathrm{cb}$.

With regard to a single reaction, mass and energy values are taken from the parent paper [50] and converted from $\mathrm{MeV}$ to $\mathrm{kg}$ (mass) and $\mathrm{J}$ (energy), $1 \mathrm{MeV}=1.78266270 \cdot 10^{-30} \mathrm{~kg}=1.60217733 \cdot 10^{-13} \mathrm{~J}$. To optimize $\Delta E_{\mathrm{ss}}$, $\Xi=\Xi^{0}(\mathrm{ssu})$ and $N=n$ are considered, as $m\left[\Xi^{-}(\mathrm{ssd})\right]$ is $7 \mathrm{MeV}$ larger than $m\left[\Xi^{0}(\mathrm{ssu})\right][50$. For further details, an interested reader is addressed to the parent paper [50], where uncertainties in $m(\Xi)$ and $\Delta E_{\mathrm{uv}}$ are also mentioned.

An inspection of Table 5 discloses total energy released from a solar mass of reagents ranges as $4.7 \cdot 10^{44} \lesssim E_{\mathrm{uv}} / \mathrm{J} \lesssim 2.2 \cdot 10^{45}$ according to quark families present in reagents. Similarly, the total energy absorbed by a solar mass of reagents in endothermic reactions amounts to $E_{\mathrm{ss}} / \mathrm{J} \approx-1.8 \cdot 10^{45}$.

On the other hand, kinetic energy released from typical supernova explosions range as $10^{43} \lesssim E_{\mathrm{SN}} / \mathrm{J} \lesssim 10^{45}, 1 \mathrm{~J}=10^{7} \mathrm{erg}$, e.g., [67] [54], and total energy released from core-collapse supernovae can grow up to a few $10^{46}$ mainly due to neutrino emission e.g., [56] 60] 39]. Accordingly, energy carried by neutrinos can safely be considered as equal to binding energy, $E_{\mathrm{B}}$, released in core collapse to form NS/QS e.g., [56] [59].

In general, the binding energy of a discrete matter distribution is defined 
as the difference in mass between matter distribution at infinite dispersion (the particle mass, $N \bar{m}$ ) and the gravitational mass (the system mass, $M$ ) e.g., 60]. In Newtonian gravity, the binding energy of a homogeneous sphere is the opposite of the potential energy e.g., [59] or in explicit form:

$$
E_{\mathrm{B}}=\zeta_{\mathrm{pot}} \frac{G M^{2}}{R}=\zeta_{\mathrm{pot}} \frac{G M^{2}}{R_{\mathrm{g}}} \frac{R_{\mathrm{g}}}{R}=\zeta_{\mathrm{pot}} \frac{G M^{2}}{2 G M c^{-2}} \frac{R_{\mathrm{g}}}{R}=\zeta_{\mathrm{pot}} \beta^{*} M c^{2}
$$

where $\zeta_{\text {pot }}$ is an increasing function of central to mean density ratio, $\rho_{\mathrm{c}} / \bar{\rho}$, and $\zeta_{\text {pot }}=3 / 5$ for a homogeneous sphere, $\bar{\rho}=\rho_{\mathrm{c}}$. The special case of spherical-symmetric polytropes reads e.g., [25] Chap. IV $\S 7$ :

$$
E_{\mathrm{B}}=\frac{3}{5-n} \beta^{*} M c^{2}
$$

ranging from homogeneous $(n=0)$ to Roche or infinitely extended $(n=5)$ configurations.

With regard to NS/QS remnants, an empirical relation inferred from EOSs consistent with an upper mass limit, $M_{\max } \gtrsim 1.65 m_{\odot}$, is [57] [59] [60]:

$$
\begin{aligned}
& \frac{E_{\mathrm{B}}}{M c^{2}}=\frac{3}{5} \beta^{*}\left(1-\frac{1}{2} \beta^{*}\right)^{-1} ; \\
& \Delta \frac{E_{\mathrm{B}}}{M c^{2}}=\frac{1}{20} \beta^{*}\left(1-\frac{1}{2} \beta^{*}\right)^{-1} ;
\end{aligned}
$$

where $0.10 \lesssim \beta^{*} \leq \beta_{\max }^{*}, \beta_{\max }^{*} \approx 0.35$ e.g., [59].

With regard to above considered NS/QS remnants, compactness parameter, $\beta^{*}$, can be inferred from Tables 2 and 4 , where $R$ and $R_{\mathrm{g}}$ are listed, respectively. Related binding energy, $E_{\mathrm{B}} /\left(M c^{2}\right)$, can be estimated via Eq. (54). Results are plotted in Fig.7 for TOV (diamonds) and EQB (squares) configurations. The tolerance, $\mp \Delta\left[E_{\mathrm{B}} /\left(M c^{2}\right)\right]$, is represented by the dashed band. Also plotted for comparison are PSR J0737-3039A (asterisk) 92] and PSR J0348+0432 (triangles) related to lower (left) and upper (right) mass limit [122].

Under the restrictive assumption NS/QS binding energy for fixed EOS depends only on compactness parameter, as:

$$
E_{\mathrm{B}}=\beta^{*} f\left(\beta^{*}\right) M c^{2} ;
$$

the classical counterpart, expressed by Eq. (52), is reproduced provided $\zeta_{\text {pot }}=$ $f\left(\beta^{*}\right)$. The special case of polytropes reads $f\left(\beta^{*}\right)=3 /(5-n)$. On the 
other hand, $f\left(\beta^{*}\right)=3 /\left[5\left(1-\beta^{*} / 2\right)\right]$ via Eq. (54a). Accordingly, compactness parameter may be related to polytropic index as:

$$
\frac{3}{5-n}=\frac{3}{5}\left(1-\frac{1}{2} \beta^{*}\right)^{-1} \quad ; \quad \frac{5-n}{5}=1-\frac{1}{2} \beta^{*} ; \quad \frac{n}{5}=\frac{1}{2} \beta^{*} ;
$$

which is equivalent to:

$$
n=\frac{5}{2} \beta^{*}
$$

and keeping in mind $0.10 \lesssim \beta^{*} \lesssim 0.35$ for NS/QS e.g., [59], related polytropic indexes read $1 / 4 \lesssim n \lesssim 7 / 8$. Classical $\left(\beta^{*}=0\right)$ and relativistic $\left(\beta^{*}=1 / 2\right)$ limit imply $n=0$ and $n=5 / 4$, respectively.

Though stable NS/QS configurations cannot exist for $\beta^{*}>\beta_{\max }^{*} \approx 0.35$ e.g., [59], binding energy extrapolation to relativistic limit could be of some interest. Using the empirical fit, $f(1 / 2)=(3 / 5) /(3 / 4)=4 / 5$ and $E_{\mathrm{B}}(1 / 2)=$ $(2 / 5) M c^{2}$ via Eqs. (54) and (55). An improved fit, based on an array of piecewise polytrope EOSs consistent with an upper mass limit, $M_{\max } \gtrsim 1.97 \mathrm{~m}_{\odot}$, and limited to $\beta \gtrsim 0.1$ [106], yields $E_{\mathrm{B}}(1 / 2)=(1 / 2)(0.564+0.521 / 2) M c^{2}=$ $0.41225 M c^{2}$. The value, $E_{\mathrm{B}}(1 / 2)=(1 / 2) M c^{2}$, would imply binding energy equals one half rest energy at $\mathrm{BH}$ limit and larger values erase baryons as distinct particles.

An empirical fit can be arranged to satisfy the above mentioned boundary condition with the addition of a new term, $E_{\mathrm{B}}^{\prime}\left(\beta^{*}\right)$, which is consistent with the tolerance of the original fit for $\beta^{*}<\beta_{\max }^{*} \approx 0.35$. Accordingly, the following relations hold:

$$
\begin{aligned}
& \frac{E_{\mathrm{B}}^{\prime}\left(\beta_{\max }^{*}\right)}{M c^{2}} \leq \Delta \frac{E_{\mathrm{B}}\left(\beta_{\max }^{*}\right)}{M c^{2}} ; \\
& \frac{E_{\mathrm{B}}(0)+E_{\mathrm{B}}^{\prime}(0)}{M c^{2}}=0 ; \\
& \frac{E_{\mathrm{B}}(1 / 2)+E_{\mathrm{B}}^{\prime}(1 / 2)}{M c^{2}}=\frac{1}{2} ;
\end{aligned}
$$

where, for reasons of simplicity, attention shall be restricted to the fit expressed by Eq. (54) and to an exponential function with negative argument for $E_{\mathrm{B}}$ and $E_{\mathrm{B}}^{\prime}$, respectively.

Under the above mentioned restrictions, the arranged fit takes the expression:

$$
\frac{E_{\mathrm{B}}\left(\beta^{*}\right)+E_{\mathrm{B}}^{\prime}\left(\beta^{*}\right)}{M c^{2}}=\left\{\frac{3}{5} \beta^{*}+\frac{3}{20} \beta^{*} \exp \left[-\kappa\left(\frac{1}{2}-\beta^{*}\right)\right]\right\}\left(1-\frac{1}{2} \beta^{*}\right)^{-1} ;
$$


accordingly, Eqs. (57) and (59) read:

$$
\begin{aligned}
& \frac{3}{20} \beta_{\max }^{*} \exp \left[-\kappa\left(\frac{1}{2}-\beta_{\max }^{*}\right)\right]\left(1-\frac{1}{2} \beta_{\max }^{*}\right)^{-1} \leq \frac{1}{20} \beta_{\max }^{*} \\
& \times\left(1-\frac{1}{2} \beta_{\max }^{*}\right)^{-1} ; \\
& \frac{E_{\mathrm{B}}(1 / 2)+E_{\mathrm{B}}^{\prime}(1 / 2)}{M c^{2}}=\left[\frac{3}{5} \frac{1}{2}+\frac{3}{20} \frac{1}{2} \exp (0)\right]\left(1-\frac{1}{2} \frac{1}{2}\right)^{-1}=\frac{15}{40} \frac{4}{3}=\frac{1}{2} ;
\end{aligned}
$$

where, setting $\beta_{\max }^{*}=0.35$, the inequality, Eq. (61), reduces to:

$\exp (-0.15 \kappa) \leq \frac{20}{3} \frac{1}{20}=\frac{1}{3} ; \quad-0.15 \kappa \leq-\ln 3 ; \quad \kappa \geq \frac{20}{3} \ln 3 \approx 7.324082 ;$

where a suitable choice reads $\kappa=8$.

Accordingly, the arranged fit via Eq. (60) takes the explicit expression:

$$
\frac{E_{\mathrm{B}}\left(\beta^{*}\right)+E_{\mathrm{B}}^{\prime}\left(\beta^{*}\right)}{M c^{2}}=\frac{3}{5} \beta^{*}\left\{1+\frac{1}{4} \exp \left[-8\left(\frac{1}{2}-\beta^{*}\right)\right]\right\}\left(1-\frac{1}{2} \beta^{*}\right)^{-1}
$$

which is plotted in Fig.7 as a full curve.

NS/QS binding energy amounts to a few $10^{46} \mathrm{~J}$ e.g., [56] [60] [39] [13], which exceeds energy released via quark-level fusion by a factor of about 10. In other words, NS/QS binding energy changes of about $10 \%$ at most during quark-level fusion, preventing either supernova explosion or collapse into submassive $\mathrm{BH}$.

\section{Discussion}

The transition from NS to BH remnants shows a desert within the mass range, $2 \lesssim M / m_{\odot} \lesssim 4$ e.g., [31] as illustrated in Fig.1]. Leaving aside occasional fallback from supernovae, massive $\left(M \gtrsim 8 m_{\odot}\right)$ ZAMS progenitors seem to be excluded for the following reasons: (i) the lower mass limit of $\mathrm{BH}$ progenitors equals $15 m_{\odot}$ and, (ii) the lower mass limit of BHs from massive progenitors equals $4 m_{\odot}$; as shown for solar metallicities in a recent investigation [93]. In this view, different processes must be considered for the formation of subHecore (in particular, submassive) BHs.

Compact remnants in close binaries, such as WD-WD, WD-NS, NS-NS, merge after orbital decay via gravitational radiation, as in the recent event GW 170817 where the inferred mass is $2.73_{-0.01}^{+0.04} m_{\odot}$ [2]. According to initial masses, the merger product could be WD, NS, subHe-core BH, or supernova explosion leaving no remnant. Binary systems hosting NS and donor MS 
star could yield submassive BH via mass accretion onto NS and subsequent instability, where $\mathrm{BH}$ presence should be inferred as mass accretion goes on. But submassive BHs in binary systems have not been detected up today.

Ultramassive, rapidly rotating or highly magnetized NSs, where spin rate or magnetic field are progressively reduced, finally collapse into submassive BHs e.g., 63] 47] [96. Energy release from quark-level reactions is found to be less than NS binding energy, $E_{\mathrm{B}} \approx 1-3 \cdot 10^{46} \mathrm{~J}$, by a factor of about 10 as shown in Table 5, which rules out the possibility of supernova explosion from slowly rotating or lowly magnetized, ultramassive NSs.

The rarity of the above mentioned events, i.e. orbital decay in close WDWD, WD-NS, NS-NS binaries; spin decrease or magnetic field attenuation in ultramassive NSs; fallback from supernovae yielding subHe-core BHs; by itself could provide an explanation to the desert between NS and $\mathrm{BH}$ remnants.

On the other hand, biases and/or selection effects cannot still be excluded, keeping in mind isolated BHs are difficult to be detected. Concerning X-ray transients with low-mass donors, it has been argued that understimates of the inclination, by $10^{\circ}$ at least, could significantly reduce $\mathrm{BH}$ mass estimates, filling the gap between the low end of $\mathrm{BH}$ mass distribution and the maximum theoretical NS mass [52].

The rarity of mildly massive $\left(4 \lesssim M / m_{\odot} \lesssim 6\right)$ BH remnants is somewhat surprising. If related ZAMS progenitors exhibit $M \gtrsim 15 m_{\odot}$ [93] and the initial mass function is decreasing with increasing mass, BH remnants would privilege the lower end of mass distribution, contrary to current data, as shown in Fig.1. The above considerations hold for isolated ZAMS progenitors, while massive stars are mainly born as close binaries, which could inhibit the formation of mildly massive $\mathrm{BH}$ remnants.

Though heavy baryons can undergo quark-level fusion [50], energy released is lower than NS binding energy by a factor of about 10, which prevents supernova explosions as in supramassive WDs. Then rapidly spinning or highly magnetized, ultramassive NSs would inescapably collapse into submassive BHs via spin or magnetic field reduction, but no clear evidence for these events has been found up today.

An upper BH mass limit of about $16 m_{\odot}$, inferred from stellar evolution 93, would imply BH-BH merger products via orbital decay in close binaries, of about $32 m_{\odot}$ at most for ZAMS progenitors of solar metallicity. On the other hand, merger products up to about $60 m_{\odot}$ have been inferred from gravitational radiation e.g., [1], contrary to the above prediction unless related ZAMS progenitors exhibit etremely low metallicity, $Z \approx 10^{-4} Z_{\odot}$ [109], or strong magnetic fields [86], as pointed out in a recent investigation [93]. 


\section{Conclusion}

The occurrence of a desert between NS and BH remnants was reviewed using a set of well-determined masses form different sources, as listed in Tables 6-13 and plotted in Fig.1. Leaving aside biases and selection effects, special effort was devoted to two specific points, namely (i) comparison between physical parameters related to NS/QS (TOV and EQB) and BH (nonrotating and maximally rotating) configurations of equal mass, respectively; and, (ii) occurrence of supernova explosions via quark-level reactions in heavy baryons, leaving no remnant.

Concerning point (i) above, NS/QS physical parameters were taken or inferred from a recent investigation [63] for 4 (NS) and 3 (QS) physically motivated EOSs, as listed in Table 2. Related BH physical parameters were determined, as listed in Table 4 .

With regard to $\eta$ - $\beta^{*}$ relation, TOV configurations were found to be consistent or marginally inconsistent with empirical fits inferred from sets of reliable EOSs [57] [39]; conversely, EQB configurations were found to be largely inconsistent (but displaced on a parallel sequence) for NSs and consistent for QSs, as shown in Fig.5.

With regard to $J-M$ relation, EQB configurations were found to be located near a straight line of similar slope in comparison to maximally rotating $\mathrm{BHs}$, but shifted downwards due to lower angular momentum by a factor of about 3.5851, as shown in Fig.6.

Concerning point (ii) above, energy released from quark-level reactions involving heavy baryons (per solar mass of reagents) was determined using results by a recent investigation [50], as listed in Table 5. Even if NSs are entirely made of heavy baryons, total energies released via above mentioned reactions were found to be still lower by a factor of about 10 with respect to binding energy, which inhibits NS supernova explosions as in supramassive WDs.

A definitive answer to the existence of a desert between NS and BH remnants is related to the following questions.

(1) Could ultramassive NSs actually collapse into submassive BHs?

(2) Could accreting NSs from MS donors in close orbits actually collapse into submassive BHs?

(3) Are there biases and/or selection effects which prevent to infer the presence of submassive BHs?

(4) Are there biases and/or selection effects which prevent to infer the presence of mildly massive BHs? 
To this respect, increasingly refined theoretical and observational techniques are needed.

\section{Acknowledgements}

This research has made use of the Exoplanet Orbit Database and the Exoplanet Data Explorer at exoplanets.org.

\section{References}

[1] B.P. Abbott, R. Abbott, T.D. Abbott, M.R. Abernathy, F. Acernese, K. Ackley, C. Adams, T. Adams, et al., Properties of the binary black hole merger GW 150914, Physical Review Letters PRL 116:241102 (2016), 19pp.

[2] B.P. Abbott, R. Abbott, T.D. Abbott, F. Acernese, K. Ackley, C. Adams, T. Adams, P. Addesso, et al., GW 170817: observation of gravitational waves from a binary neutron star inspiral, Physical Review Letters PRL 119:161101 (2017), 18pp.

[3] J. Abedi, N. Afshordi, Echoes from the abyss: a highly spinning black hole remnant from the binary neutron star merger GW170817, arXiv:1803.10454 (2018), 14pp.

[4] S. Ai, H. Gao, Z.-G. Dai, X.-F. Wu, A. Li, B. Zhang, M.-Z. Li, The allowed parameter space of a long-lived neutron star as the merger remnant of GW 170817, The Astrophysical Journal 860:57 (2018), 9pp.

[5] J. Alsing, H.O. Silva, E. Berti, Evidence for a maximum mass cut-off in the neutron star mass distribution and constraints on the equation of state, Monthly Notices of the Royal Astronomical Society 478 (2018), 1377-1391.

[6] E. Andriani, R. Caimmi, On the properties of elliptical and spiral galaxies in a CDM scenario, Astrophysics and Space Science 168 (1990), 131-152.

[7] J. Antoniadis, M.H. van Kerkwijk, D. Koester, P.C.C. Freire, N. Wex, T.M. Tauris, M. Kramer, C.G. Bassa, The relativistic pulsar-white dwarf binary PSR J1738+0333 - I. Mass determination and evolutionary history, Monthly Notices of the Royal Astronomical Society 423 (2012), 3316-3327.

[8] J. Antoniadis, P.C.C. Freire, N. Wex, T.M. Tauris, R.S. Lynch, M.H. van Kerkwijk, M. Kramer, C.G. Bassa, V.S. Dhillon, T. Driebe, et al., 
A massive pulsar in a compact relativistic binary, Science 340:1233232 (2013), 9pp.

[9] J. Antoniadis, T.M. Tauris, F. Ozel, E. Barr, D.J. Champion, P.C.C. Freire, The millisecond pulsar mass distribution: evidence for bimodality and constraints on the maximum neutron star mass, arXiv:1605.01665 (2016), 21pp.

[10] S. Balberg, S.L. Shapiro, The properties of matter in white dwarfs and neutron stars, arxiv:astro-ph/0004317v2 (2000), 31pp.

[11] N.D.R. Bhat, M. Bayles, J.P.W. Verbiest, Gravitational-radiation losses from the pulsar white dwarf binary PSR J1141 6545, Physical Review D $77: 124017$ (2008), 5pp.

[12] P. Bodenheimer, G. D'Angelo, J.J. Lissauer, J.J. Fortney, D. Saumon, Deuterium burning in massive giant planets and low-mass brown dwarfs formed by core-nucleated accretion, The Astrophysical Journal 770:120 (2013), 13pp.

[13] I. Bombaci, D. Logoteta, Equation of state of dense nuclear matter and neutron star structure from nuclear chiral interactions, Astronomy \& Astrophysics 609:A128 (2018), 19pp.

[14] H.E. Bond, R.L. Gilliand, G.H. Schaefer, P. Demarque, T.M. Girard, J.B. Holberg, D. Gudehus, B.D. Mason, et al., Hubble Space Telescope astrometry of the Procyon system, The Astrophysical Journal 813:106 (2015), 19pp.

[15] H.E. Bond, G.H. Schaefer, R.L. Gilliand, J.B. Holberg, B.D. Mason, I.W. Lindenblad, M. Seitz-McLeese, W.D. Arnett, et al., The Sirius system and its astrophysical puzzles: Hubble Space Telescope and ground-based astrometry, The Astrophysical Journal 840:70 (2017), 17pp.

[16] M.C.P. Bours, T.R. Marsh, S.G. Parsons, C.M. Copperwheat, V.S. Dhillon, S.P. Littlefair, B.T. Gänsicke, A. Gianninas, P.-E. Tremblay, Precise parameters for both white dwarfs in the eclipsing binary CSS 41177, Monthly Notices of the Royal Astronomical Society 438 (2014), 3399-3408.

[17] A.J. Burgasser, Brown dwarfs: failed stars, super Jupiters, Physics Today 61 (2008), 70-71.

[18] V.D. Burkert, L. Elouadrhiri, F.X. Girod, The pressure distribution inside the proton, Nature 557 (2018), 396-399. 
[19] B. Burningham, Large-scale searches for brown dwarfs and free-floating planets, arXiv:1805.05661 (2018), 26pp.

[20] A. Burrows, M.S. Marley, W.B. Hubbard, J.I. Lunine, T. Guillot, D. Saumon, R. Freedman, D. Sudarsky, C. Sharp, A nongray theory of extrasolar giant planets and brown dwarfs, The Astrophysical Journal 491 (1997), 856-875.

[21] A. Burrows, D. Sudarsky, C. Sharp, M.S. Marley, W.B. Hubbard, J.I. Lunine, T. Guillot, D. Saumon, R. Freedman, Advances in the theory of brown dwarfs and extrasolar giant planets, ASP Conference Series 134 (1997) eds. R. Rebolo, E.L. Martin, M.R. Zapatero Osorio, 1998, 354-369.

[22] A. Burrows, W.B. Hubbard, J.I. Lunine, J. Liebert, The theory of brown dwarfs and extrasolar giant planets, Reviews of Modern Physics 73 (2001), 719-765.

[23] A.D. Cameron, D.J. Champion, M. Kramer, M. Bailes, E.D. Barr, C.G. Bassa, S. Bhandari, N.D.R. Bhat, M. Burgay, S. Burke-Spolaor, et al., The high time resolution universe pulsar survey - XIII. PSR J1757-1854, the most accelerated binary pulsar, Monthly Notices of the Royal Astronomical Society 475 (2018), L57-L61.

[24] C.I. Cañas, C.F. Bender, S. Mahadevan, S.W. Fleming, T.G. Beatty, K.R. Covey, N. De Lee, F.R. Hearty, et al., Kepler-503b: an object at the hydrogen burning mass limit orbiting a subgiant star, The Astrophysical Journal 861:L4 (2018), 7pp.

[25] S. Chandrasekhar, An introduction to the study of the stellar structure, Dover Publications, Inc., New York, unabridged University of Chicago Press, 1939.

[26] U. Das, B. Mukhopadhyay, Maximum mass of stable magnetized highly super-Chandrasekhar white dwarfs: stable solutions with varying magnetic fields, Journal of Cosmology and Astroparticle Physics 6:50 (2014), 10pp.

[27] G. Desvignes, R.N. Caballero, L. Lentati, J.P.W. Verbiest, D.J. Champion, B.W. Stappers, G.H. Janssen, P. Lazarus, S. Osłowski, S. Babak, et al., High-precision timing of 42 millisecond pulsars with the European pulsar timing array, Monthly Notices of the Royal Astronomical Society 458 (2016), 3341-3380. 
[28] S.B. Dieterich, A.J. Weinberger, A.P. Boss, T.J. Henry, W.-C. Jao, J. Gagné, T.L. Astraatmadja, M.A. Thompson, G. Anglada-Escudé, Dynamical masses of $\epsilon$ Indi B and C: two massive brown dwarfs at the edge of the stellar-substellar boundary, The Astrophysical Journal 865:28 (2018), $19 \mathrm{pp}$.

[29] Exoplanet Orbit Database and Exoplanet Data Explorer, exoplanets.org/table (consulted on June 2018).

[30] M. Falanga, E. Bozzo, A. Lutovinov, J.M. Bonnet-Bidaud, Y. Fetisova, J. Puls, Ephemeris, orbital decay, and masses of ten eclipsing high-mass X-ray binaries, Astronomy \& Astrophysics 577:A130 (2015), 16pp.

[31] W.H. Farr, N. Sravan, A. Cantrell, L. Kreidberg, C.D. Bailyn, I. Mandel, V. Kalogera, The mass distribution of stellar-mass black holes, The Astrophysical Journal 741:103 (2011), 19pp.

[32] R.D. Ferdman, I.H. Stairs, M. Kramer, M.A. McLaughlin, D.R. Lorimer, D.J. Nice, R.N. Manchester, G. Hobbs, A.G. Lyne, F. Camilo, et al., A precise mass mesaurement of the intermediate-mass binary pulsar PSR J1802-2124, The Astrophysical Journal 711 (2010), 764-771.

[33] R.D. Ferdman, I.H. Stairs, M. Kramer, G.H. Janssen, C.G. Bassa, B.W. Stappers, P.B. Demorest, I. Cognard, G. Desvignes, G. Theureau, et al., PSR J1756-2251: a pulsar with a low-mass neutron star companion, Monthly Notices of the Royal Astronomical Society 443 (2014), 2183-2196.

[34] E. Fonseca, I.H. Stairs, S.E. Thorsett, A comprehensive study of relativistic gravity using PSR B1534+12, The Astrophysical Journal 787:82 (2014), 9pp.

[35] E. Fonseca, T.T. Pennucci, J.A. Ellis, I.H. Stairs, D.J. Nice, S.M. Ransom, P.B. Demorest, Z. Arzoumanian, K. Crowter, T. Dolch, et al., The NANOGrav nine-year data set: mass and geometric mesaurements of binary millisecond pulsars, The Astrophysical Journal 832:167 (2016), 22pp.

[36] J.C. Forbes, A. Loeb, On the existence of brown dwarfs more massive than the hydrogen burning limit, arXiv:1805.12143 (2018), 11pp.

[37] P.C.C. Freire, C.G. Bassa, N. Wex, I.H. Stairs, D.J. Champion, S.M. Ransom, P. Lazarus, V.M. Kaspi, J.W.T. Hessels, M. Kramer, et al., On the nature and evolution of the unique binary pulsar J1903+0327, Monthly Notices of the Royal Astronomical Society 412 (2011), 2763-2780. 
[38] G. Gagnè, G. Fontaine, A. Simon, J.K. Faherty, A young ultramassive white dwarf in the AB Doradus moving group, The Astrophysical Journal 861:L13 (2018), 6pp.

[39] A. Gallo Rosso, F. Vissani, M.C. Volpe, Measuring the neutron star compactness and binding energy with supernova neutrinos, Journal of Cosmology and Astroparticle Physics 11 (2017) 036, 12pp.

[40] D.M. Gelino, T.E. Harrison, GRO J0422+32: the lowest mass black hole?, The Astrophysical Journal 599 (2003), 1254-1259.

[41] N. Giammichele, S. Carpinet, G. Fontaine, P. Brassard, E.M. Green, V. Van Grootel, P. Bergeron, W. Zong, M.-A. Dupret, A large oxygendominated core from the seismic cartography of a pulsating white dwarf, Nature 554 (2018), 73-76.

[42] B. Giesers, S. Dreizler, T.-O. Husser, S. Kamann, G. Anglada Escudé, J. Brinchmann, C.M. Carollo, M.M. Roth, P.M. Weilbacher, L. Wisotzki, A detached stellar-mass black hole candidate in the globular cluster NGC 3201, Monthly Notices of the Royal Astronomical Society 475 (2018), L15L19.

[43] J. Girven, B.T. Gänsicke, B. Külebi, D. Steeghs, S. Jordan, T.R. Marsh, D. Koester, PG1258+593 and its common proper motion magnetic white dwarf counterpart, Monthly Notices of the Royal Astronomical Society 404 (2010), 159-165.

[44] J.I. González Hernández, R. Rebolo, J. Casares, Fast orbital decays of black hole X-ray binaries: XTE J1118+480 and A0620-00, Monthly Notices of the Royal Astronomical Society 438 (2014), L21-L25.

[45] B. Greene, The elegant universe, W.W. Norton \& Company, New York, 1999.

[46] J. Greiner, J.G. Cuby, M.J. McCaughrean, An unusually massive stellar black hole in the Galaxy, Nature 414 (2001), 522-525.

[47] P. Haensel, M. Bejger, M. Fortin, L. Zdunik, Rotating neutron stars with exotic cores: masses, radii, stability, The European Physical Journal A 52:59 (2016), 21pp.

[48] V. Hodzic, H.M.J. Triaud, D.R. Anderson, F. Bouchy, A. Collier Cameron, L. Delrez, M. Gillon, C. Ellier, et al., WASP-128b: a transiting brown dwarf in the dynamical-tide regime, Monthly Notices of the Royal Astronomical Society 481 (2018), 5091-5097. 
[49] B.A. Jacoby, P.B. Cameron, F.A. Jenet, S.B. Anderson, R.N. Murty, S.R. Kulcarni, Mesaurement of orbital decay in the double neutron star binary PSR B2127+11C, The Astrophysical Journal 644 (2006), L113L116.

[50] M. Karliner, J.L. Rosner, Quark-level analogue of nuclear fusion with doubly heavy baryons, Nature 551 (2017), 89-91.

[51] M. Kramer, I.H. Stairs, R.N. Manchester, M.A. McLaughlin, A.G. Lyne, L.D. Ferdman, M. Burgay, D.R. Lorimer, A. Possenti, N. D'Amico, et al., Tests of general relativity from timing the double pulsar, Science 314 (2006), 97-102.

[52] L. Kreidberg, C.D. Bailyn, W.M. Farr, V. Kalogera, Mass mesaurements of black holes in X-ray transients: is there a mass gap?, The Astrophysical Journal 757:36 (2012), 17pp.

[53] A. Kundu, B. Mukhopadhyay, Mass of Highly Magnetized White Dwarfs Exceeding the Chandrasekhar Limit: An Analytical View, Modern Physics Letters A 27:1250084 (2012), 15pp.

[54] D. Kushnir, Thermonuclear explosion of rotating massive stars could explain core-collapse supernovae, arXiv:1502.03111 (2015), 11pp.

[55] L. Landau, E. Lifchitz, Theorie du champs, Mir, Moscow, 1966.

[56] J.M. Lattimer, A. Yahil, Analysis of the neutrino events from supernova 1987A, The Astrophysical Journal 340 (1989), 426-434.

[57] J.M. Lattimer, M. Prakash, Neutron star structure and the equation of state, The Astrophysical Journal 550 (2001), 426-442.

[58] J.M. Lattimer, M. Prakash, The physics of neutron stars, Science 304 (2004), 536-542.

[59] J.M. Lattimer, M. Prakash, Neutron star observations: prognosis for equation of state constraints, Physics Reports 442 (2007), 109-165.

[60] J.M. Lattimer, M. Prakash, The equation of state of hot, dense matter and neutron stars, Physics Reports 621 (2016), 127-164.

[61] N.M. Law, A.L. Kraus, R. Street, B.J. Fulton, L.A. Hillenbrand, A. Sphorer, T. Lister, C. Baranec, J.S. Bloom, K. Bui, et al., Three new eclipsing white-dwarf-M-dwarf binaries discovered in a search for transiting planets around M-dwarfs, The Astrophysical Journal 757:133 (2012), 14pp. 
[62] P.F. Lazorenko, J. Sahlmann, Updated astrometry and masses of the LUH 16 brown dwarf binary, Astronomy \& Astrophysics 618:A111 (2018), $11 \mathrm{pp}$.

[63] A. Li, B. Zhang, N.-B. Zhang, H. Gao, B. Qi, T. Liu, Internal Xray plateau in short GRBs: signature of supramassive fast-rotating quark stars?, Physical Review D 94:083010 (2016), 6pp.

[64] R.S. Lynch, P.C.C. Freire, S.M. Ransom, B.A. Jacoby, The timing of nine globular clusters pulsars, The Astrophysical Journal 745:109 (2012), $12 \mathrm{pp}$.

[65] J.G. Martinez, K. Stovall, P.C.C. Freire, J.S. Deneva, F.A. Jenet, M.A. McLaughlin, M. Bagchi, S.D. Bates, A. Ridolfi, Pulsar J0453+1559: a double neutron star system with a large mass asymmetry, The Astrophysical Journal 812:143 (2015), 8pp.

[66] B.D. Mason, W.I. Hartkopf, K.N. Miles, Binary star orbits. V. The nearby white dwarf / red dwarf pair 40 Eri BC, The Astronomical Journal 154:200 (2017), 9pp.

[67] P.A. Mazzali, F.K. Röpke, S. Benetti, W. Hillebrandt, A common explosion mechanism for type Ia supernovae, Science 315 (2007), 825-828.

[68] S. Mereghetti, A. Tiengo, P. Esposito, N. La Palombara, G.L. Israel, L. Stella, An ultramassive, fast-spinning white dwarf in a peculiar binary system, Science 325 (2009), 1222-1223.

[69] G.A. Miller, Quark fuse to release energy, Nature 551 (2017), 40-41.

[70] M.S. O'Brien, H.E. Bond, E.M. Sion, Hubble Space Telescope spectroscopy of V471 Tauri: oversized K star, paradoxical white dwarf, The Astrophysical Journal 563 (2001), 971-986.

[71] J.A. Orosz, J.E. McClintock, R. Narayan, C.D. Baylin, J.D. Hartman, L. Macri, J. Liu, W. Pietsch, R.A. Remillard, A. Shporer, T. Mazeh, A 15.65-solar mass black hole in an eclipsing binary in the nearby spiral galaxy M33, Nature 449 (2007), 872-875.

[72] J.A. Orosz, D. Steeghs, J.E. McClintock, M.A.P. Torres, I. Bochkov, L. Gou, R. Narayan, M. Blaschak, A.M. Levine, R.A. Remillard, et al., A new dynamical model for the black hole binary LMC X-1, The Astrophysical Journal 697:75 (2011), 13pp. 
[73] J.A. Orosz, J.F. Steiner, J.E. McClintock, M.A.P. Torres, R.A. Remillard, C.D. Baylin, J.M. Miller, An improved model for the microquasar XTE J1550-564, The Astrophysical Journal 730 (2009), 573-591.

[74] J.A. Orosz, Inventory of black hole binaries, A massive star odyssey, from main sequence to supernova, Proceedings IAU Symposium 212 (2003) IAU, K.A. van der Hutch, A. Herrero, C. Esteban, eds., 365-371.

[75] J.P. Ostriker, P. Bodenheimer, D. Lynden-Bell, Equilibrium Models of Differentially Rotating Zero-Temperature Stars, Physical Review Letters, 17 (1966), 816-818.

[76] J. P. Ostriker, P. Bodenheimer, Rapidly rotating stars. II. Massive white dwarfs, The Astrophysical Journal 151 (1968), 1089-1098.

[77] F. Özel, D. Psaltis, R. Narayan, E. McClintock, The black hole mass distribution in the Galaxy, The Astrophysical Journal 725 (2010), 19181927.

[78] F. Özel, P. Freire, Masses, radii, and the equation of state of neutron stars, Annual Review of Astronomy \& Astrophysics 54 (2016), 401-440.

[79] S.G. Parsons, T.R. Marsh, C.M. Copperwheat, V.S. Dhillon, S.P. Littlefair, B.T. Gänsicke, R.D.G. Hickman, Precise mass and radius values for the white dwarf and low mass $M$ dwarf in the pre-cataclysmic binary NN Serpentis, Monthly Notices of the Royal Astronomical Society 402 (2010), 2591-2608.

[80] S.G. Parsons, T.R. Marsh, B.T. Gänsicke, A. Rebassa-Mansergas, V.S. Dhillon, S.P. Littlefair, C.M. Copperwheat, R.D.G. Hickman, et al., A precision study of two eclipsing white dwarf plus M dwarf binaries, Monthly Notices of the Royal Astronomical Society 420 (2012), 3281-3297.

[81] S.G. Parsons, B.T. Gänsicke, T.R. Marsh, P. Bergeron, C.M. Copperwheat, V.S. Dhillon, J. Bento, S.P. Littlefair, M.R. Schreiber, An accurate mass and radius mesaurement for an ultracool white dwarf, Monthly Notices of the Royal Astronomical Society 426 (2012), 1950-1958.

[82] S.G. Parsons, T.R. Marsh, B.T. Gänsicke, V.S. Dhillon, C.M. Copperwheat, S.P. Littlefair, S. Pyrzas, A.J. Drake, et al., The shortest period detached white dwarf + main sequence binary, Monthly Notices of the Royal Astronomical Society 419 (2012), 304-313. 
[83] S.G. Parsons, C.A. Hill, T.R. Marsh, B.T. Gänsicke, C.A. Watson, D. Steeghs, V.S. Dhillon, S.P. Littlefair, et al., The crowded magnetosphere of the post-common envelope binary QS Virginis, Monthly Notices of the Royal Astronomical Society 458 (2016), 2793-2812.

[84] S.G. Parsons, B.T. Gänsicke, T.R. Marsh, R.P. Ashley, M.C.P. Bours, E. Breedt, M.R. Burleigh, C.M. Copperwheat, et al., Testing the white dwarf mass-radius relationship with eclipsing binaries, Monthly Notices of the Royal Astronomical Society 470 (2017), 4473-4492.

[85] S. Peretti, D. Ségransan, B. Lavie, S. Desidera, A.-L. Maire, V. D’Orazi, A. Vigan, J.-L. Baudino, A. Cheetham, et al., Orbital and spectral analysis of the benchmark brown dwarf HD 4747B, arXiv:1805.05645 (2018), 21pp.

[86] V. Petit, Z. Kesztheliy, R. MacInnis, D.H. Cohen, R.H.D. Townsend, G.A. Wade, S.L. Thomas, S.P. Owocki, et al., Magnetic massive stars as progenitors of 'heavy' stellar-mass black holes, Monthly Notices of the Royal Academic Society 466 (2017), 1052-1060.

[87] M. Piotrovich, Y. Gnedin, T. Natsvlishvili, S. Buliga, Estimates of supermassive black holes (SMBH) spins for the standard accretion disk model: comparison with relativistic fitting of SMBH spectra, New Astronomy (2018), 25-28.

[88] C. Providência, M. Fortin, H. Pais, A. Rabhi, Hyperonic stars and the symmetry energy, arXiv:1811.00786 (2018), 17pp.

[89] S. Pyrzas, B.T. Gänsicke, S. Brady, S.G. Parsons, T.R. Marsh, D. Koester, E. Breedt, C.M. Copperwheat, et al., Post-common envelope binaries from SDSS - XV. Accurate stellar parameters for a cool $0.4 M_{\odot}$ white dwarf and a $0.16 M_{\odot} \mathrm{M}$ dwarf in a $3 \mathrm{~h}$ eclipsing binary, Monthly Notices of the Royal Astronomical Society 419 (2012), 817-826.

[90] A.R. Raduta, A. Sedrakian, F. Weber, Cooling of hypernuclear compact stars, Monthly Notices of the Royal Astronomical Society 475 (2018), 43474356.

[91] C.A. Raithel, F. Özel, D. Psaltis, From neutron star observables to the equation of state. I. An optimal parametrization, The Astrophysical Journal 831:44 (2016), 11pp.

[92] C.A. Raithel, F. Özel, D. Psaltis, Model-independent inference of neutron star radii from moment of inertia mesaurements, Physical Review $\mathrm{C}$ 93:032801 (R) (2016), 4pp. 
[93] C.A. Raithel, T. Sukhbold, F. Özel, Confronting models of massive star evolution and explosions with remnant mass mesaurements, The Astrophysical Journal 856:35 (2018), 13pp.

[94] S.M. Ransom, I.H. Stairs, A.M. Archibald, J.W.T. Hessels, D.L. Kaplan, M.H. van Kerkwijk, J. Boyles, A.T. Deller, S. Chatterjee, A. Schechtman, et al., A millisecond pulsar in a stellar triple system, Nature 505 (2014), 520-524.

[95] D.J. Reardon, G. Hobbs, W. Coles, Y. Levin, M.J. Keith, M. Bailes, N.D.R. Bhat, S. Burke-Spolaor, S. Dai, M. Kerr, et al., Timing analysis for 20 millisecond pulsars in the Parkes Pulsar Timing Array, Monthly Notices of the Royal Astronomical Society 455 (2016), 1751-1769.

[96] L. Rezzolla, E.R. Most, L.R. Weih, Using gravitational-wave observations and quasi-universal relations to constrain the maximum mass of neutron stars, The Astrophysical Journal 852:L25 (2018), 5pp.

[97] V.V. Sagun, I. Lopes, Neutron stars: a novel equation of state with induced surface tension, The Astrophysical Journal 850:75 (2017), 6pp.

[98] V.V. Sagun, I. Lopes, A.I. Ivanytskyi, The induced surface tension contribution for the equation of state of neutron stars, arXiv:1805.04976 (2018), 7pp.

[99] K.C. Sahu, J. Anderson, S. Casertano, H.E. Bond, P. Bergeron, E.P. Nelan, L. Pueyo, T.M. Brown, et al., Relativistic deflection of background starlight measures the mass of a nearby white dwarf star, Science 356 (2017), 1046-1050.

[100] K.C. Schlaufman, Evidence of an upper bound on the masses of planets and its implications for giant planet formation, The Astrophysical Journal 853:37 (2018), 14pp.

[101] N.I. Shakura, G.V. Lipunova, Logarithmic potential for the gravitational field of Schwartzschild black holes, Monthly Notices of the Royal Astronomical Society, 480 (2018), 4273-4277.

[102] M. Sieniawska, M. Bejger, B. Haskell, Estimating the equation of state from mesaurements of neutron star radii with $5 \%$ accuracy, Astronomy \& Astrophysics 616:A105 (2018), 9pp.

[103] D.S. Spiegel, A. Burrows, J.A. Milsom, The deuterium-burning mass limit for brown dwarfs and giant planets, The Astrophysical Journal 727:57 (2011), 9pp. 
[104] A.W. Steiner, J.M. Lattimer, E.F. Brown, The equation of state from observed masses and radii of neutron stars, The Astrophysical Journal 722 (2010), 33-54.

[105] A.W. Steiner, J.M. Lattimer, E.F. Brown, The neutron star massradius relation and the equation of state of dense matter, The Astrophysical Journal 765:L5 (2013), 5pp.

[106] A.V. Steiner, J.M. Lattimer, E.F. Brown, Neutron star radii, universal relations, and the role of prior distributions, The European Physical Journal A 52:18 (2016), 16pp.

[107] K. Stovall, P.C.C. Freire, J. Antoniadis, M. Bagchi, J.S. Deneva, N. Garver-Daniels, J.G. Martinez, M.A. McLaughlin, et al., PSR J2234+0611: a new laboratory for stellar evolution, arXiv:1809.05064 (2018), 16pp.

[108] J.P. Subasavage, W.-C. Jao, T.J. Henry, H.C. Harris, C.C. Dahn, P. Bergeron, P. Dufour, B.H. Dunlap, et al., The solar neighbourhood. XXXIX. Parallax results from the CTIOPI and NOFS programs: 50 new members of the 25 parsec white dwarf sample, The Astronomical Journal 154:32 (2017), 24pp.

[109] T. Sukhbold, S.E. Woosley, The compactness of presupernova stellar cores, The Astrophysical Journal 783:10 (2014), 20pp.

[110] K.S. Thorne, Disk-accretion onto a black hole. II. Evolution of the hole, The Astrophysical Journal 191 (1974), 507-520.

[111] E.P.J. van den Heuvel, Double neutron stars: evidence for two different neutron-star formation mechanisms, AIP Conference Proceedings 924 (2007), 598-606.

[112] M.H. van Kerkwijk, S.R. Kulkarni, A massive white dwarf companion to the eccentric binary pulsar system PSR B2303+46, The Astrophysical Journal 516 (1999), L25-L28.

[113] J. van Leeuwen, L. Kasian, I.H. Stairs, D.R. Lorimer, F. Camilo, S. Chatterjee, I. Cognard, G. Desvignes, P.C.C. Freire, G.H. Janssen, et al., The binary companion of young, relativistic pulsar J1906+0746, The Astrophysical Journal 798:118 (2015), 15pp.

[114] J.-B. Wei, A. Figura, G.F. Burgio, H. Chen, H.-J. Schulze, Neutron star universal relations with microscopic equations of state, arXiv:1809.04315 (2018), 16pp. 
[115] J.M. Weiswberg, D.J. Nice, J.H. Taylor, Timing mesaurements of the relativistic binary pulsar PSR B1913+16, The Astrophysical Journal 722 (2010), 1030-1034.

[116] J. Wu, J.A. Orosz, J.E. McClintock, I. Hasan, C.D. Baylin, L. Gou, Z. Chen, The mass of the black hole in the X-ray binary NOVA MUSCAE 1991, The Astrophysical Journal 825:46 (2016), 13pp.

[117] K. Yagi, N. Yunes, I-Love-Q relations in neutron stars and their applications to astrophysics, gravitational waves, and fundamental physics, Physical Review D 88:023009 (2013), 28pp.

[118] K. Yagi, N. Yunes, Approximate universal relations for neutron stars and quark stars, Physics Reports 681 (2017), 1-72.

[119] Z.H. Zhang, D.J. Pinfield, M.C. Gálvez-Ortiz, B. Burningham, N. Lodieu, F. Marocco, A.J. Burgasser, A.C. Day-Jones, F. Allard, H.R.A. Jones, et al., Primeval very low-mass stars and brown dwarfs - I. Six new L subdwarfs, classification and atmospheric properties, Monthly Notices of the Royal Astronomical Society 464 (2017), 3040-3059.

[120] Z.H. Zhang, D. Homeier, D.J. Pinfield, N. Lodieu, H.R.A. Jones, F. Allard, Ya. V. Pavlenko, Primeval very low-mass stars and brown dwarfs - II. The most metal-poor substellar object, Monthly Notices of the Royal Astronomical Society 468 (2017), 261-271.

[121] Z.H. Zhang, D.J. Pinfield, M.C. Gálvez-Ortiz, D. Homeier, A.J. Burgasser, N. Lodieu, E.L. Martín, M.R. Zapatero Osorio, F. Allard, H.R.A. Jones, R.L. Smart, B. López Martí, B. Burningham, R. Rebolo, Primeval very low-mass stars and brown dwarfs - III. The halo transitional brown dwarfs, Monthly Notices of the Royal Astronomical Society 479 (2018), 1383-1391.

[122] X.-F. Zhao, The moment of inertia of the neutron star PSR J0348+0432 and its proto neutron star, Astrophysics and Space Science 362:95 (2017), 4pp.

[123] E. Zhou, A. Tsokaros, L. Rezzolla, R. Xu, Maximum mass of axisymmetric rotating quark stars, Astronomische Nachrichten 338 (2017), 10441047.

[124] W.W. Zhu, I.H. Stairs, P.B. Demorest, D.J. Nice, J.A. Ellis, S.M. Ransom, Z. Arzoumanian, K. Crowter, T. Dolch, R.D. Ferdman, et al., Testing theories of gravitation using 21-year timing of pulsar binary J1713+0747, The Astrophysical Journal 809:41 (2015), 15pp. 


\section{Appendix}

\section{A Masses of stellar remnants}

Stellar remnants with well determined masses i.e. percent error $\Delta^{\%} m=$ $100(\Delta M / M)<10$ or $\Delta M / M<0.1$, shown in Fig.1, are listed below in Tables 6, 7, 8, 9, 11, 12, 13, for BDs, MS dwarfs, WDs, NSs, BHs, respectively. Owing to the paucity of known $\mathrm{BH}$ remnants at present, data affected by larger percent error are also listed in Table 13 . 
Table 6: High-precision $(\Delta M / M<0.1)$ masses and uncertainties for $N=41$ brown dwarf (BD) stars hosted in binary systems. Masses are in solar units.

\begin{tabular}{lllll}
\hline \multicolumn{1}{c}{ system } & \multicolumn{1}{c}{$M$} & $+\Delta M$ & $-\Delta M$ & ref \\
\hline CoRoT-3 b & 0.02081 & 0.00095 & 0.00095 & {$[29]$} \\
CoRoT-15 & 0.0604 & 0.0039 & 0.0039 & {$[100]$} \\
CoRoT-27 b & 0.00990 & 0.00073 & 0.00073 & {$[29]$} \\
CoRoT-33 & 0.056 & 0.0016 & 0.0017 & {$[100]$} \\
gamma Leo A b & 0.00990 & 0.00079 & 0.00079 & {$[29]$} \\
EPIC 201702477 & 0.0639 & 0.0016 & 0.0016 & {$[100]$} \\
EPIC 219388192 & 0.0348 & 0.00009 & 0.00009 & {$[100]$} \\
epsilon Indi B & 0.0716 & 0.0008 & 0.0008 & {$[28]$} \\
epsilon Indi C & 0.0669 & 0.00064 & 0.00064 & {$[28]$} \\
LUH 16 A & 0.03199 & 0.00030 & 0.00028 & {$[62]$} \\
LUH 16 B & 0.02725 & 0.00025 & 0.00024 & {$[62]$} \\
HAT-P-13 c & 0.01362 & 0.00066 & 0.00066 & {$[29]$} \\
HD 4747 B & 0.0668 & 0.0015 & 0.0015 & {$[85]$} \\
HD 217786 b & 0.0126 & 0.00109 & 0.00109 & {$[29]$} \\
HD 168443 c & 0.01660 & 0.00055 & 0.00055 & {$[29]$} \\
HD 97233 b & 0.0189 & 0.00142 & 0.00142 & {$[29]$} \\
HD 162020 b & 0.01452 & 0.00049 & 0.00049 & {$[29]$} \\
HD 22781 b & 0.01321 & 0.00047 & 0.00047 & {$[29]$} \\
HD 180314 b & 0.0216 & 0.00168 & 0.00168 & {$[29]$} \\
HD 202206 b & 0.01606 & 0.00065 & 0.00065 & {$[29]$} \\
HD 16760 b & 0.01269 & 0.000153 & 0.000153 & {$[29]$} \\
HD 131664 b & 0.0175 & 0.00124 & 0.00124 & {$[29]$} \\
HD 38529 c & 0.01170 & 0.0004 & 0.0004 & {$[29]$} \\
HD 136118 b & 0.1115 & 0.0004 & 0.0004 & {$[29]$} \\
HD 38801 b & 0.00956 & 0.00041 & 0.00041 & {$[29]$} \\
HD 39091 b & 0.00963 & 0.00036 & 0.00036 & {$[29]$} \\
HD 106270 b & 0.01058 & 0.00083 & 0.00083 & {$[29]$} \\
HD 156846 b & 0.01051 & 0.00036 & 0.00036 & {$[29]$} \\
HD 114762 b & 0.01111 & 0.00074 & 0.00074 & {$[29]$} \\
HD 10069 & 0.00970 & 0.00035 & 0.00035 & {$[100$} \\
KELT-1 & 0.02613 & 0.00088 & 0.00088 & {$[100]$} \\
Kepler-39 b & 0.01735 & 0.00069 & 0.00069 & {$[29]$} \\
KOI-205 & 0.0389 & 0.0014 & 0.0011 & {$[100]$} \\
KOI-415 & 0.05932 & 0.00257 & 0.00257 & {$[100]$} \\
TYC 3727-1064-1 & 0.01247 & 0.00062 & 0.00062 & {$[100]$} \\
WASP-18 b & 0.00961 & 0.00032 & 0.00032 & {$[29]$} \\
WASP-30 & 0.0597 & 0.0012 & 0.0012 & {$[100]$} \\
\hline
\end{tabular}


Table 7: High-precision $(\Delta M / M<0.1)$ masses and uncertainties for $N=41$ brown dwarf (BD) stars hosted in binary systems. Masses are in solar units. Continuation of Table 6.

\begin{tabular}{lllll}
\hline \multicolumn{1}{c}{ system } & \multicolumn{1}{c}{$M$} & $+\Delta M$ & $-\Delta M$ & ref \\
\hline WASP-128 b & 0.0358 & 0.0008 & 0.0008 & {$[48$} \\
XO-3 b & 0.01246 & 0.00042 & 0.00042 & {$[29]$} \\
10 Del b & 0.00983 & 0.00034 & 0.00034 & {$[29]$} \\
11 Com b & 0.0154 & 0.00147 & 0.00147 & {$[29]$} \\
\hline
\end{tabular}

Table 8: High-precision $(\Delta M / M<0.1)$ masses and uncertainties for $N=19$ main sequence (MS) dwarf stars hosted in binary systems. Masses are in solar units.

\begin{tabular}{lllll}
\hline \multicolumn{1}{c}{ system } & $M$ & $+\Delta M$ & $-\Delta M$ & ref \\
\hline EBLM J0555-57A & 0.0813 & 0.0037 & 0.0038 & {$[100$} \\
HATS550-016 & 0.1098 & 0.0057 & 0.0048 & {$[100]$} \\
HAT-TR-205-013 & 0.124 & 0.0095 & 0.0095 & {$[100]$} \\
Kepler-503 b & 0.075 & 0.003 & 0.003 & {$[24]$} \\
KIC 1571511 & 0.143 & 0.0038 & 0.0048 & {$[100$} \\
KOI-189 & 0.074 & 0.0032 & 0.0032 & {$[100]$} \\
KOI-686 & 0.0987 & 0.0046 & 0.0046 & {$[100]$} \\
OGLE-TR-122 & 0.092 & 0.0086 & 0.0086 & {$[100]$} \\
SDSS J010448.46+153501.8 & 0.0860 & 0.0015 & 0.0015 & {$[120]$} \\
SDSS J125637.13-022452.4 & 0.0833 & 0.0015 & 0.0015 & {$[119]$} \\
ULAS J020858.62+020657.0 & 0.0827 & 0.0015 & 0.0015 & {$[121]$} \\
ULAS J135058.86+081506.8 & 0.0833 & 0.0015 & 0.0015 & {$[121]$} \\
ULAS J151913.03-000030.0 & 0.0811 & 0.0015 & 0.0015 & {$[119]$} \\
ULAS J230711.01+014447.1 & 0.0822 & 0.0015 & 0.0015 & {$[121$} \\
TYC 7760-484-1 & 0.0911 & 0.0024 & 0.0018 & {$[100$} \\
1SWASPJ234318.41+295556.5 & 0.0955 & 0.0067 & 0.0067 & {$[100$} \\
2MASS J05325346+8246465 & 0.0802 & 0.0015 & 0.0015 & {$[119]$} \\
2MASS J06164006-6407194 & 0.0805 & 0.0015 & 0.0015 & {$[119]$} \\
2MASS J16262034+3925190 & 0.0828 & 0.0015 & 0.0015 & {$[119]$} \\
\hline
\end{tabular}


Table 9: High-precision $(\Delta M / M<0.1)$ masses and uncertainties for $N=51$ white dwarf (WD) stars hosted in binary systems and $N=25$ nearby stars belonging to the 25-pc WD sample [108]. Masses are in solar units.

\begin{tabular}{|c|c|c|c|c|}
\hline system & $M$ & $+\Delta M$ & $-\Delta M$ & ref \\
\hline CSS 080502 & 0.4756 & 0.0036 & 0.0036 & 84 \\
\hline CSS 09704 & 0.4164 & 0.0356 & 0.0356 & 84 \\
\hline CSS 21357 & 0.6579 & 0.0097 & 0.0097 & 84 \\
\hline CSS 40190 & 0.4817 & 0.0077 & 0.0077 & 84 \\
\hline CSS 41177 A & 0.3780 & 0.0230 & 0.0230 & 16 \\
\hline CSS 41177 B & 0.3160 & 0.0110 & 0.0110 & 16 \\
\hline GD 50 & 1.28 & 0.02 & 0.02 & 38 \\
\hline GK Vir & 0.5618 & 0.0142 & 0.0142 & 80 \\
\hline KIC 08626021 & 0.570 & 0.005 & 0.005 & 41 \\
\hline NN Ser & 0.5354 & 0.0117 & 0.0117 & 79] \\
\hline PG1258+593 & 0.54 & 0.01 & 0.01 & 43 \\
\hline Procyon B & 0.592 & 0.006 & 0.006 & [14] \\
\hline PSR B1855+09 & 0.238 & 0.013 & 0.012 & 35 \\
\hline PSR B2303+46 & 1.3 & 0.1 & 0.1 & 112 \\
\hline PSR J0337+1715 A & 0.19751 & 0.00015 & 0.00015 & 94 \\
\hline PSR J0337+1715 B & 0.4101 & 0.0003 & 0.0003 & 94 \\
\hline PSR J0348+0432 & 0.172 & 0.003 & 0.003 & [8] \\
\hline PSR J0437+4715 & 0.224 & 0.007 & 0.007 & 95 \\
\hline PSR J0751+1807 & 0.16 & 0.01 & 0.01 & [27] \\
\hline PSR J1141-6545 & 1.02 & 0.01 & 0.01 & [11] \\
\hline PSR J1145-6545 & 1.00 & 0.02 & 0.02 & 111 \\
\hline PSR J1614-2230 & 0.493 & 0.003 & 0.003 & 35 \\
\hline PSR J1713+0747 & 0.286 & 0.012 & 0.012 & 124 \\
\hline PSR J1730+0333 & 0.181 & 0.007 & 0.005 & 7 \\
\hline PSR J1802-2124 & 0.78 & 0.04 & 0.04 & 32 \\
\hline PSR J1909-3744 & 0.214 & 0.003 & 0.003 & 35 \\
\hline PSR J1918-0642 & 0.219 & 0.012 & 0.011 & 35 \\
\hline PSR J2234+0611 B & 0.298 & 0.015 & 0.012 & 107 \\
\hline PTFEB11.441 & 0.54 & 0.05 & 0.05 & 61 \\
\hline QS Vir & 0.7816 & 0.0130 & 0.0130 & 83 \\
\hline RR Cae & 0.4475 & 0.0015 & 0.0015 & 84] \\
\hline
\end{tabular}


Table 10: High-precision $(\Delta M / M<0.1)$ masses and uncertainties for $N=$ 51 white dwarf (WD) stars hosted in binary systems, and $N=25$ nearby stars belonging to the 25-pc WD sample [108]. Masses are in solar units. Continuation of Table 9 .

\begin{tabular}{lllll}
\hline \multicolumn{1}{c}{ system } & $M$ & $+\Delta M$ & $-\Delta M$ & ref \\
\hline RX J0648.0-4418 & 1.28 & 0.05 & 0.05 & {$[68]$} \\
SDSS J0024+1745 & 0.5340 & 0.0090 & 0.0090 & {$[84]$} \\
SDSS J0106-0014 & 0.4406 & 0.0144 & 0.0144 & {$[84]$} \\
SDSS J0110+1326 & 0.4656 & 0.0091 & 0.0091 & {$[84]$} \\
SDSS J0138-0016 & 0.5290 & 0.0100 & 0.0100 & {$[81]$} \\
SDSS J0314+0206 & 0.5964 & 0.0088 & 0.0088 & {$[84]$} \\
SDSS J0857+0342 & 0.5140 & 0.0490 & 0.0490 & {$[82]$} \\
SDSS J1021+1744 & 0.5338 & 0.0038 & 0.0038 & {$[84]$} \\
SDSS J1028+0931 & 0.4146 & 0.0036 & 0.0036 & {$[84]$} \\
SDSS J1123-1155 & 0.6050 & 0.0079 & 0.0079 & {$[84]$} \\
SDSS J1210+3347 & 0.4150 & 0.0100 & 0.0100 & {$[89]$} \\
SDSS J1212-0123 & 0.4393 & 0.0022 & 0.0022 & {$[80]$} \\
SDSS J1307+2156 & 0.6098 & 0.0031 & 0.0031 & {$[84]$} \\
SDSS J1329+1230 & 0.3916 & 0.0234 & 0.0234 & {$[84]$} \\
SDSS J2235+1428 & 0.3977 & 0.0220 & 0.0220 & {$[84]$} \\
Sirius B & 1.018 & 0.011 & 0.011 & {$[15]$} \\
Stein 2051 B & 0.675 & 0.051 & 0.051 & {$[99]$} \\
V471 Tau & 0.8400 & 0.0500 & 0.0500 & {$[70]$} \\
WD 0000-345 & 0.68 & 0.03 & 0.03 & {$[108]$} \\
WD 0034-602 & 0.95 & 0.04 & 0.04 & {$[108]$} \\
WD 0046+051 & 0.67 & 0.02 & 0.02 & {$[108]$} \\
WD 0136+152 & 0.66 & 0.02 & 0.02 & {$[108]$} \\
WD 0243-026 & 0.73 & 0.03 & 0.03 & {$[108]$} \\
WD 0310-688 & 0.65 & 0.04 & 0.04 & {$[108]$} \\
WD 0311-649 & 0.29 & 0.02 & 0.02 & {$[108]$} \\
WD 0423+044 & 0.72 & 0.04 & 0.04 & {$[108]$} \\
WD 0457-004 & 1.10 & 0.01 & 0.01 & {$[108$} \\
WD 0511+079 & 0.84 & 0.03 & 0.03 & {$[108$} \\
WD 0548-001 & 0.70 & 0.02 & 0.02 & {$[108]$} \\
WD 0552-041 & 0.90 & 0.02 & 0.02 & {$[108]$} \\
WD 0644+025 & 0.99 & 0.01 & 0.01 & {$[108$} \\
WD 0659-063 & 0.63 & 0.03 & 0.03 & {$[108$} \\
WD 0738-172 & 0.61 & 0.03 & 0.03 & {$[108]$} \\
WD 0816-310 & 0.71 & 0.06 & 0.06 & {$[108]$} \\
\hline
\end{tabular}


Table 11: High-precision $(\Delta M / M<0.1)$ masses and uncertainties for $N=$ 51 white dwarf (WD) stars hosted in binary systems, and $N=25$ nearby stars belonging to the 25-pc WD sample [108. Masses are in solar units. Continuation of Table 10 .

\begin{tabular}{lllll}
\hline \multicolumn{1}{c}{ system } & $M$ & $+\Delta M$ & $-\Delta M$ & ref \\
\hline WD 0856-007 & 0.64 & 0.03 & 0.03 & {$[108$} \\
WD 1036-204 & 0.78 & 0.03 & 0.03 & {$[108$} \\
WD 1105-340 & 0.69 & 0.04 & 0.04 & {$[108$} \\
WD 1236-495 & 0.98 & 0.02 & 0.02 & {$[108]$} \\
WD 1333+005 & 0.4356 & 0.0016 & 0.0016 & {$[84$} \\
WD 2047+372 & 0.82 & 0.02 & 0.02 & {$[108$} \\
WD 2159-754 & 0.97 & 0.04 & 0.04 & {$[108$} \\
WD 2211-392 & 0.80 & 0.04 & 0.04 & {$[108$} \\
WD 2341+322 & 0.62 & 0.01 & 0.01 & {$[108$} \\
WD 2359-434 & 0.83 & 0.02 & 0.02 & {$[108$} \\
40 Eri B & 0.573 & 0.018 & 0.018 & {$[66]$} \\
\hline
\end{tabular}




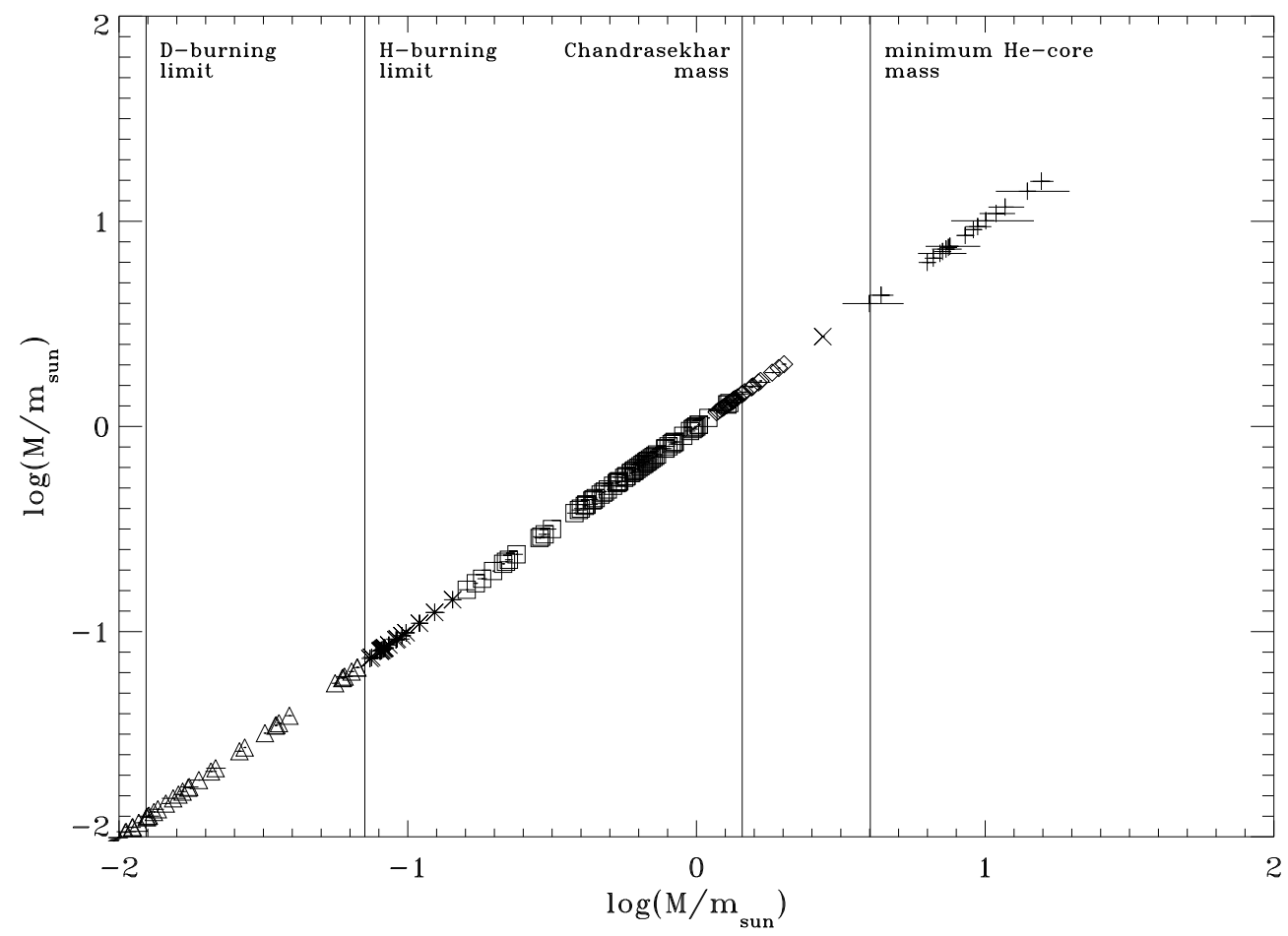

Figure 1: Brown dwarf (triangles), main sequence-dwarf (asterisks), white dwarf (squares), neutron star (diamonds), black hole (crosses) mass range for stars listed in Tables 6, 7, 8, 9, 11, 12, 13, respectively, shown as a logarithmic mass-to-mass relation in solar units. The post-merger product from the double neutron star binary GW 170817 is shown as a saltire. To avoid confusion, errors are represented as horizontal bars instead of squares. D-burning limit $\left(13 M_{\mathrm{J}}=0.01241 m_{\odot}\right)$, H-burning limit $\left(0.071 m_{\odot}\right)$, Chandrasekhar mass $\left(1.44 m_{\odot}\right)$, and minimum He-core mass $\left(4 m_{\odot}\right)$ of $\mathrm{BH}$ progenitors are marked as vertical lines. 


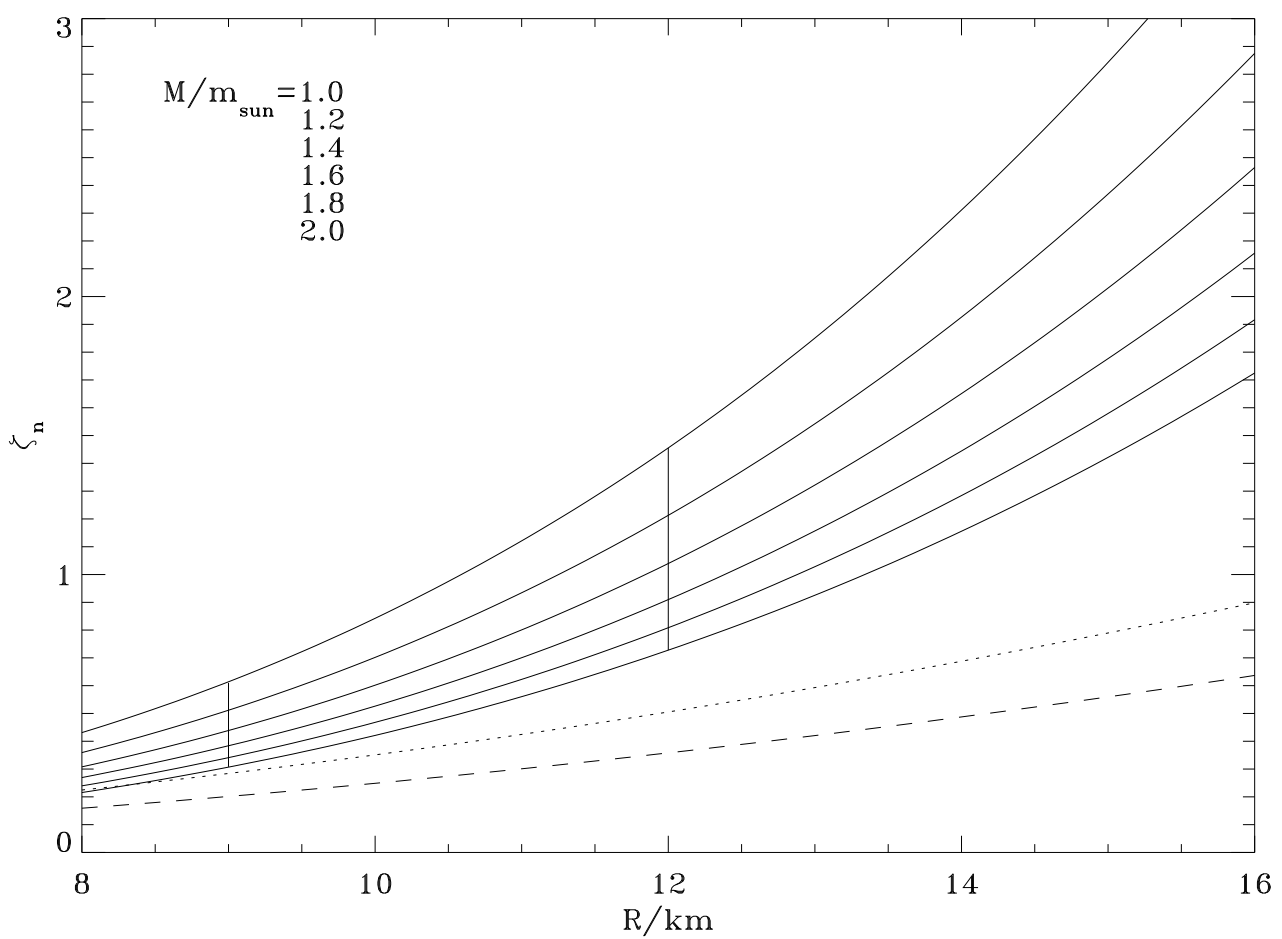

Figure 2: Neutron star density factor, $\zeta_{n}$, vs radius, $R / \mathrm{km}$, for assigned mass, $M / m_{\odot}$. Most of currently available or inferred data lie within a region bounded by $R / \mathrm{km}=9,12$, and $M / m_{\odot}=1,2$. Onset of inescapable gravitational collapse and casual relationship is marked as a dashed and dotted line, respectively. 


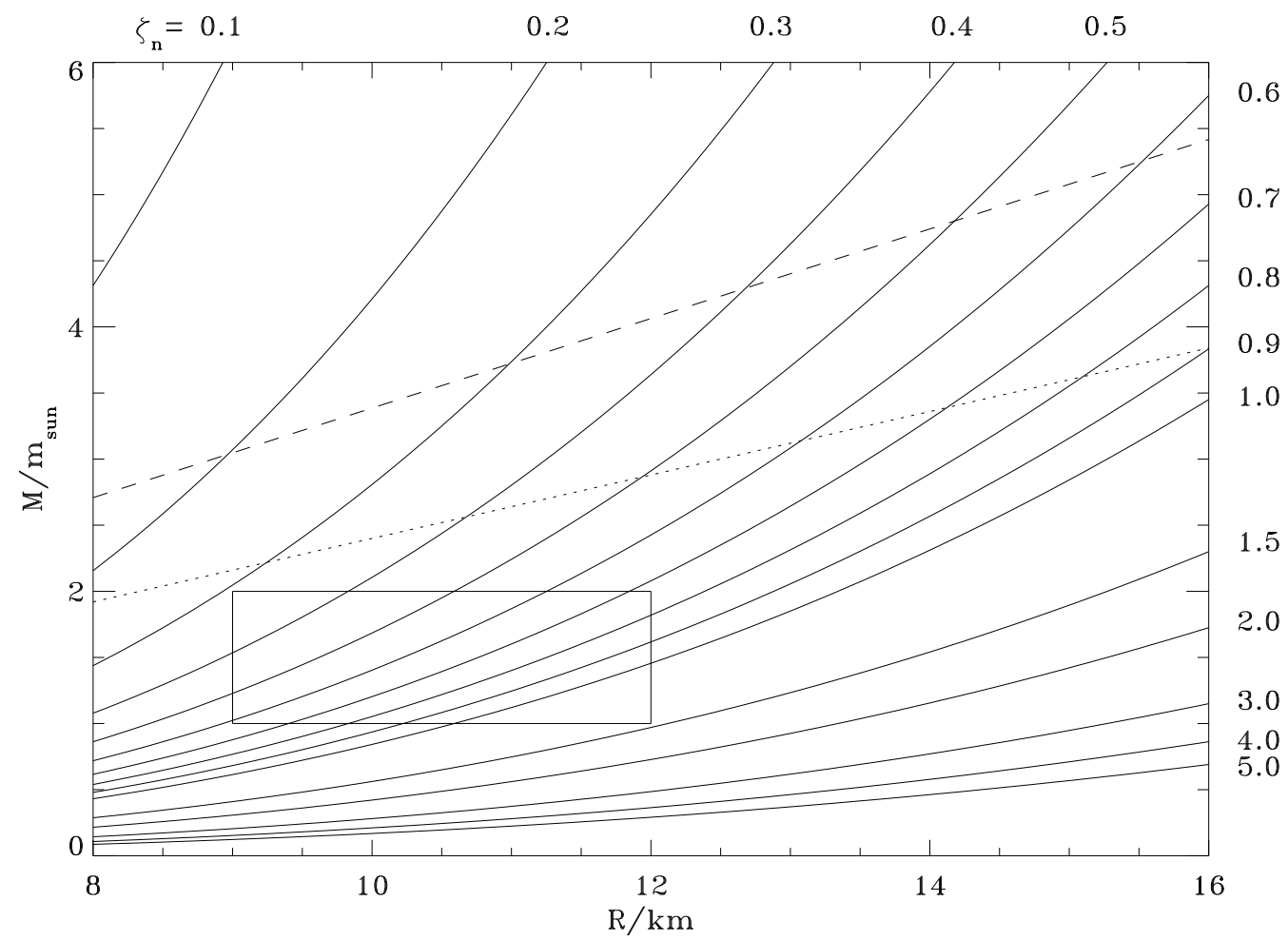

Figure 3: Neutron star mass, $M / m_{\odot}$, vs radius, $R / \mathrm{km}$, for assigned density factor, $\zeta_{n}$. Most of currently available or inferred data lie within a region bounded by $R / \mathrm{km}=9,12$, and $M / m_{\odot}=1,2$. Onset of inescapable gravitational collapse and casual relationship is marked as a dashed and dotted line, respectively. 


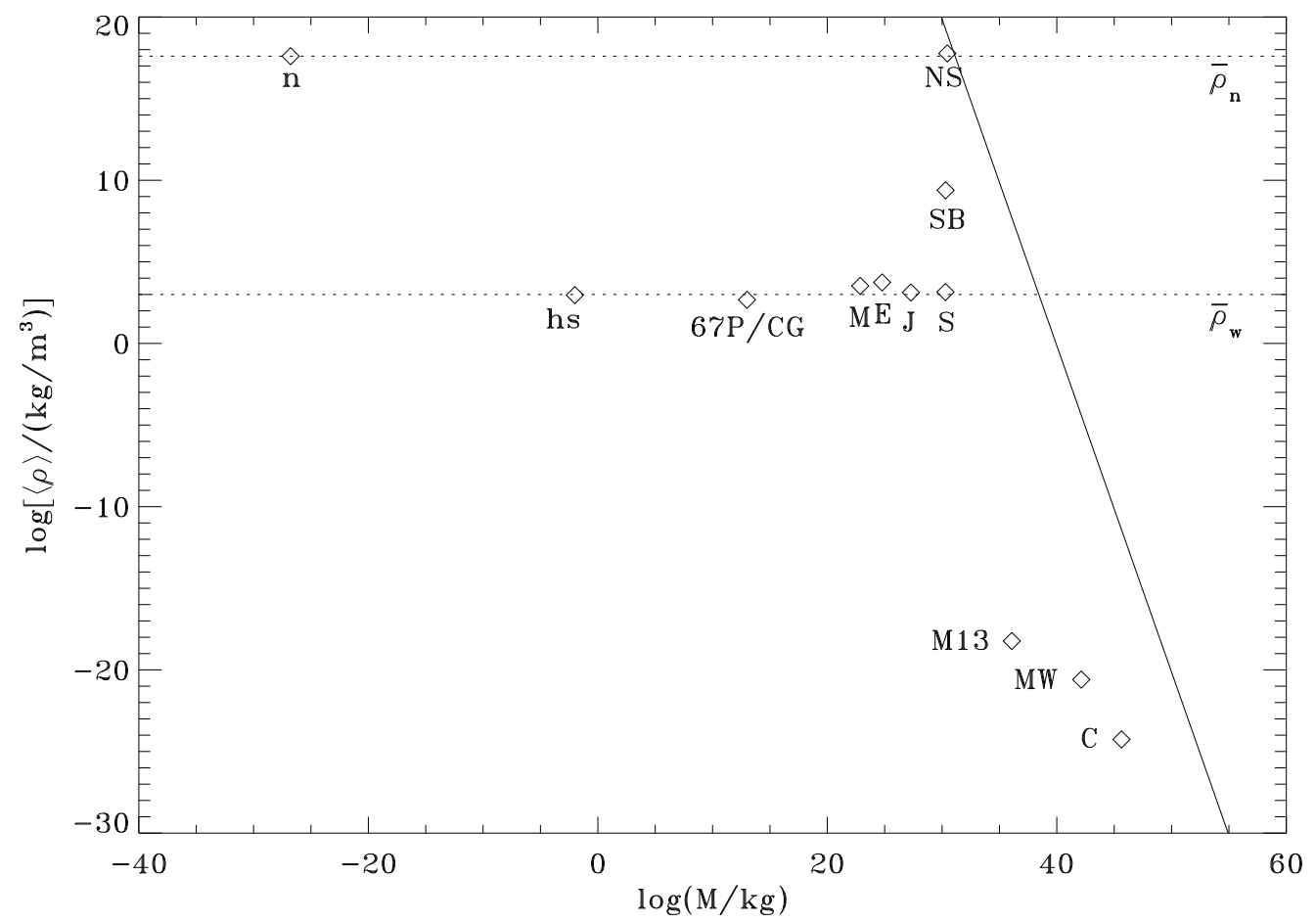

Figure 4: Mean density vs mass in the logarithmic plane for the following bodies: neutron (n); hail bead (hb); comet (67P/Churyumov-Gerasimenko, 67P/CG); Moon (M); Earth (E); Jupiter (J); Sun (S); white dwarf (Syrius $\mathrm{B}, \mathrm{SB}$ ); neutron star (PSR J0337+1715, assumed radius, NS); globular cluster (M13, M13); galaxy (Milky Way, MW); galaxy cluster (Coma, C). The threshold of inescapable gravitational collapse, $R_{\mathrm{s}}=R_{\mathrm{g}}$, is shown as a full line. Neutron mean density and water mean density are marked as dotted horizontal lines. 


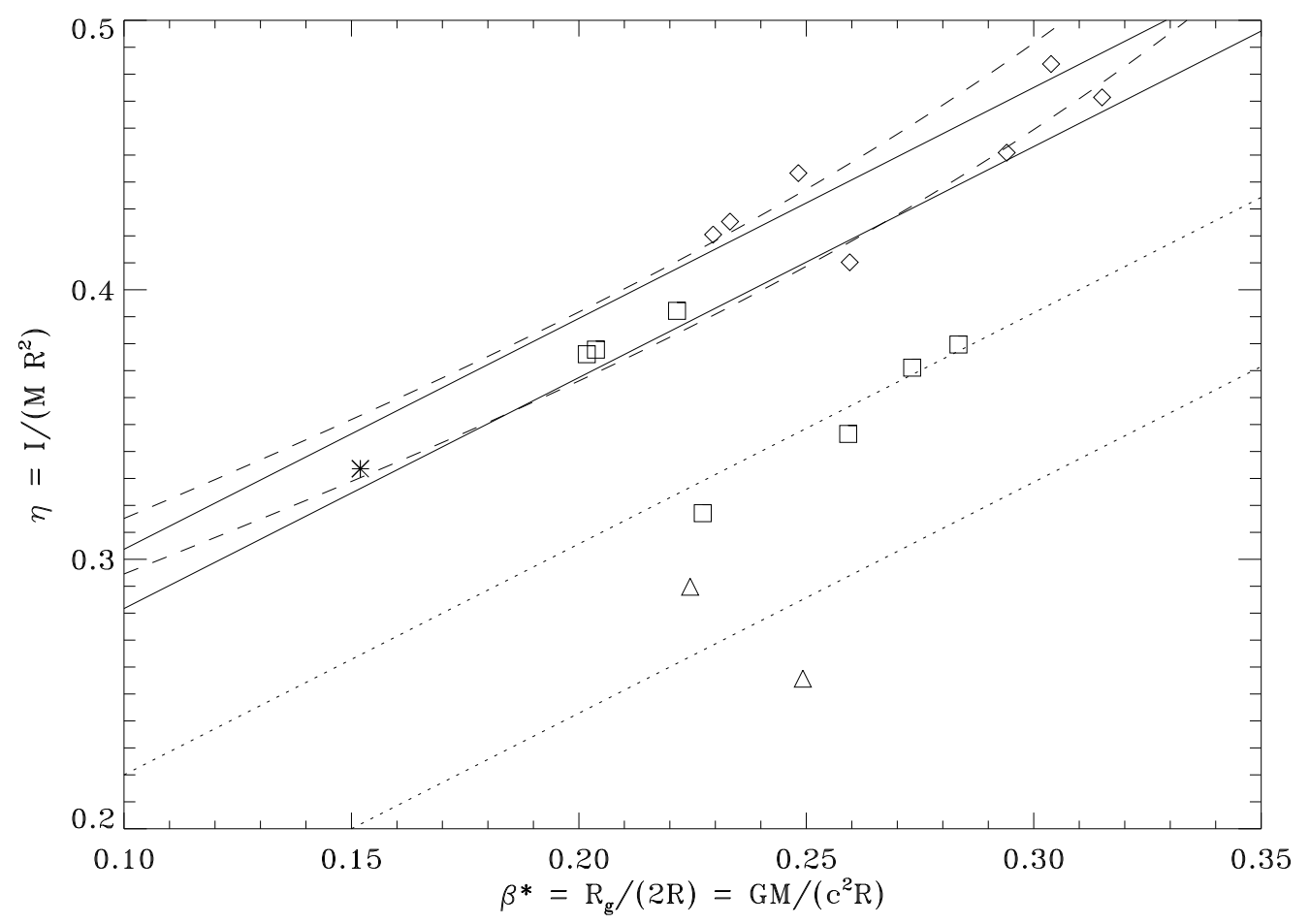

Figure 5: Profile parameter, $\eta$, vs compactness parameter, $\beta^{*}$, for TOV (diamonds) and EQB (squares) configurations listed in Table 2. Also plotted for comparison are PSR J0737-3039A (asterisk), inferred from fiducial and consistent values of $I$ and $R$ [92], and PSR J0348+0432 (triangles) related to lower (left) and upper (right) mass limit [122]. Empirical fits from different EOS sets are shown as a dashed [57] 104] 105] and full [114] band, respectively. Dotted lines are parallel to the full band and related intercepts read 0.1343 (selected for comparison with lower EQB configurations) and 0.0715 [selected for fitting to $\mathrm{BH}$ limit, $\left(\beta^{*}, \eta\right)=(1 / 2,1 / 2)$ ]. See text for further details. 


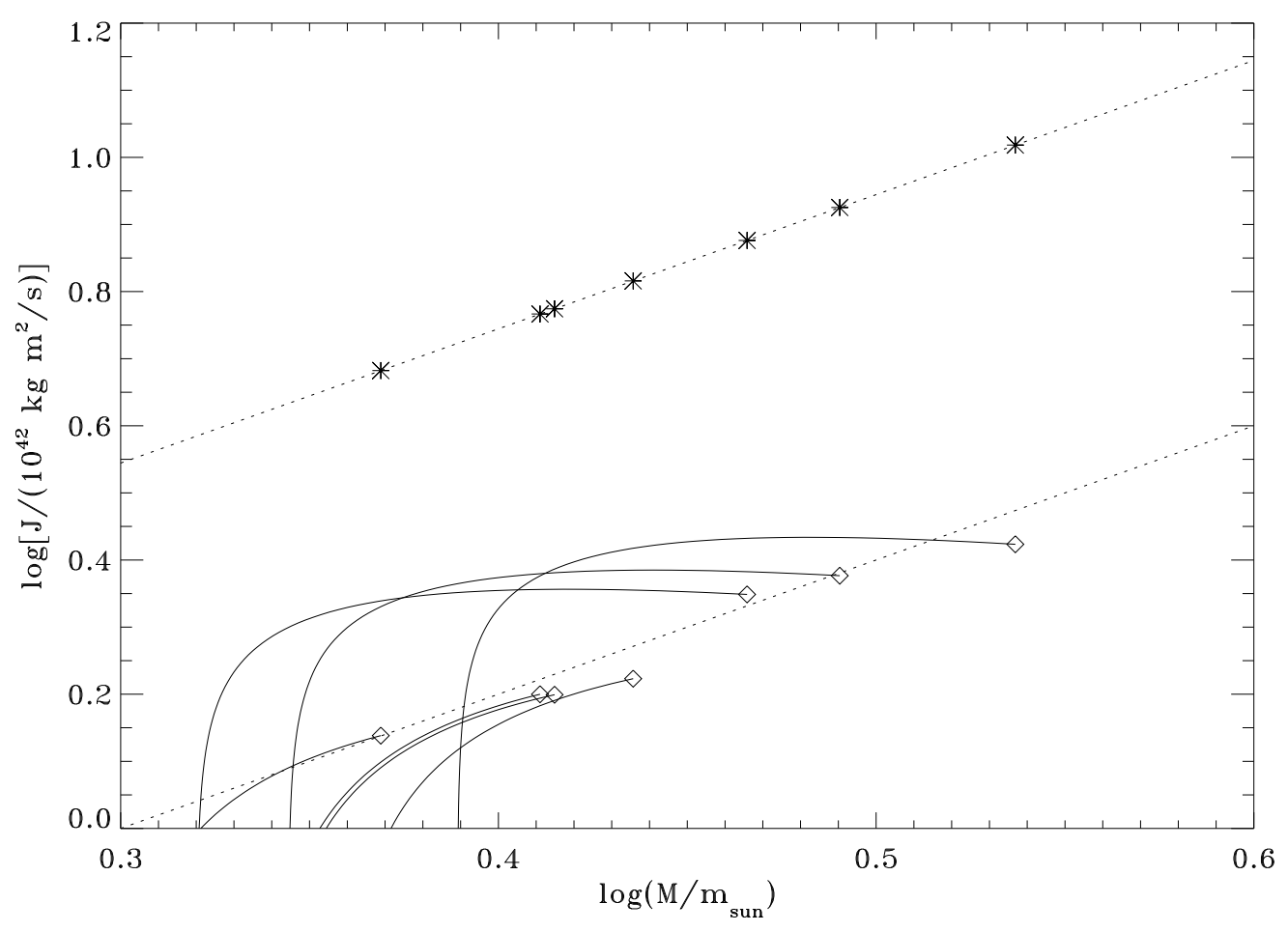

Figure 6: $J-M$ relation in logarithmic plane for EQB configurations related to NSs/QSs (diamonds) and maximally rotating BHs of equal mass, $M_{\mathrm{BH}}=M_{\mathrm{EQB}}$ (asterisks), placed on the dotted straight line of slope, 2, and intercept, -0.05545 . A parallel line (arbitrarily chosen at $\log a_{\mathrm{BH}}^{*}=-0.5545$ or $\left.a_{\mathrm{BH}}^{*}=0.2789\right)$ is placed downwards to facilitate comparison with EQB configurations. Loci of maximum mass configurations for frequencies up to EQB value are shown as full curves. With regard to EQB configurations, EOSs are, from the left to the right: (a) NSs - BCPM, BSk20, Shen, BSk21; (b) QSs - CIDDM, CDMM1, CDMM2. See text for further details. 


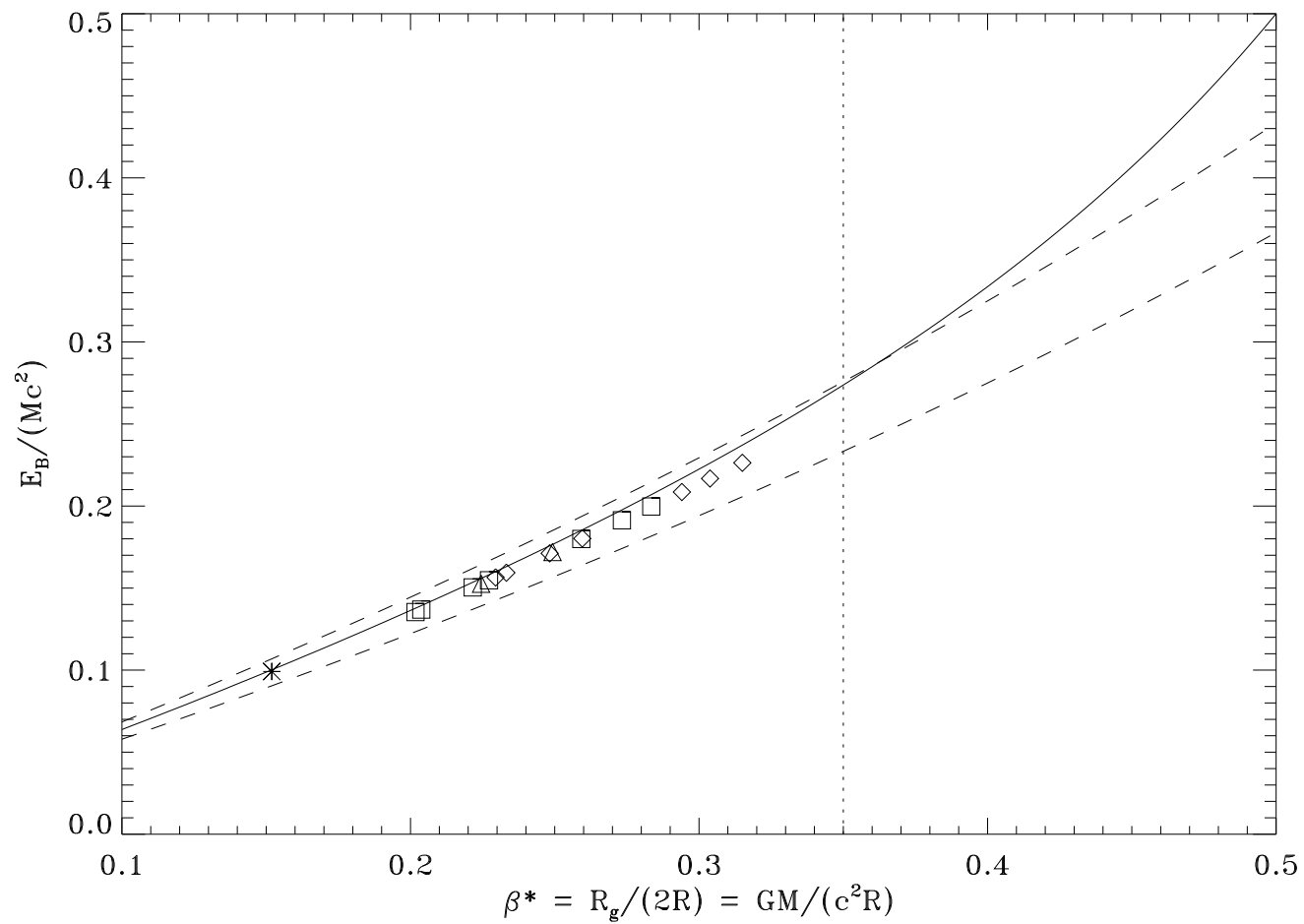

Figure 7: Binding energy of TOV (diamonds) and EQB (squares) configurations, expressed via an empirical relation, $E_{\mathrm{B}} /\left(M c^{2}\right)=(0.6 \mp 0.05) \beta^{*}(1-$ $\left.0.5 \beta^{*}\right)^{-1}$ [57], where the tolerance is marked by the dashed curves. Also plotted for comparison are PSR J0737-3039A (asterisk) 92], and PSR J0348+0432 (triangles) related to lower (left) and upper (right) mass limit [122]. The full curve results from the addition of an extra term, $\left(E_{\mathrm{B}}+\right.$ $\left.E_{\mathrm{B}}^{\prime}\right) /\left(M c^{2}\right)=\left\{0.6 \beta^{*}+0.075 \exp \left[-8\left(0.5-\beta^{*}\right)\right]\right\}\left(1-0.5 \beta^{*}\right)^{-1}$, which satisfies the conditon, $\left(E_{\mathrm{B}}+E_{\mathrm{B}}^{\prime}\right) /\left(M c^{2}\right)=0.5$, in the relativistic limit, $\beta^{*}=0.5$. The maximum compactness parameter for NS/QS, taken as $\beta_{\max }^{*}=0.35$ e.g., [59], is marked as a vertical dotted line. See text for further details. 
Table 12: High-precision $(\Delta M / M<0.1)$ masses and uncertainties for $N=$ 37 neutron star (NS) members in binary systems. Masses are in solar units.

\begin{tabular}{|c|c|c|c|c|}
\hline system & $M$ & $+\Delta M$ & $-\Delta M$ & ref \\
\hline LMC X-4 & 1.57 & 0.11 & 0.11 & 30 \\
\hline PSR B1534+12 p & 1.3330 & 0.0004 & 0.0004 & 34 \\
\hline PSR B1534+12 c & 1.3455 & 0.0004 & 0.0004 & 34 \\
\hline PSR B1855+09 & 1.31 & 0.12 & 0.10 & 35 \\
\hline PSR B1913+16 & 1.4398 & 0.0002 & 0.0002 & 115 \\
\hline PSR B1913+16 & 1.3886 & 0.0002 & 0.0002 & 115 \\
\hline PSR B2127+11 p & 1.358 & 0.010 & 0.010 & 49 \\
\hline PSR B2127+11 c & 1.354 & 0.010 & 0.010 & 49] \\
\hline PSR B2303+46 & 1.34 & 0.10 & 0.10 & 112 \\
\hline PSR J0337+1715 & 1.4378 & 0.0013 & 0.0013 & 94 \\
\hline PSR J0348+0432 & 2.01 & 0.04 & 0.04 & 8 \\
\hline PSR J0437+4715 & 1.44 & 0.07 & 0.07 & 95 \\
\hline PSR J0453+1559 p & 1.559 & 0.005 & 0.005 & 65 \\
\hline PSR J0453+1559 c & 1.174 & 0.004 & 0.004 & 65 \\
\hline PSR J0737-3039 A & 1.3381 & 0.007 & 0.007 & 51 \\
\hline PSR J0737-3039 B & 1.2489 & 0.007 & 0.007 & 51 \\
\hline PSR J0751+1807 & 1.64 & 0.15 & 0.15 & 27 \\
\hline PSR J1012+5307 & 1.83 & 0.11 & 0.11 & 9 \\
\hline PSR J1141-6545 & 1.27 & 0.01 & 0.01 & 11 \\
\hline PSR J1145-6545 & 1.28 & 0.02 & 0.02 & 111 \\
\hline PSR J1614-2230 & 1.928 & 0.017 & 0.017 & 35 \\
\hline PSR J1713+0747 & 1.31 & 0.11 & 0.11 & 124 \\
\hline PSR J1738+0333 & 1.47 & 0.07 & 0.06 & 7 \\
\hline PSR J1756-2251 p & 1.341 & 0.007 & 0.007 & 33 \\
\hline PSR J1756-2251 c & 1.230 & 0.007 & 0.007 & 33 \\
\hline PSR J1757-1854 p & 1.3384 & 0.0009 & 0.0009 & 23 \\
\hline PSR J1757-1854 c & 1.3946 & 0.0009 & 0.0009 & 23 \\
\hline PSR J1802-2124 & 1.24 & 0.11 & 0.11 & 32 \\
\hline PSR J1807-2500B & 1.3655 & 0.021 & 0.021 & 64 \\
\hline PSR J1829+2456 p & 1.30 & 0.05 & 0.05 & 111 \\
\hline PSR J1829+2456 c & 1.27 & 0.11 & 0.07 & 111 \\
\hline PSR J1903+0327 & 1.667 & 0.021 & 0.021 & 37 \\
\hline PSR J1906+0746 & 1.291 & 0.011 & 0.011 & [113 \\
\hline PSR J1909-3744 & 1.55 & 0.03 & 0.03 & 35 \\
\hline PSR J1918-0642 & 1.19 & 0.10 & 0.09 & 35 \\
\hline PSR J2234+0611 A & 1.353 & 0.014 & 0.017 & 107 \\
\hline SMC X-1 & 1.21 & 0.12 & 0.12 & 30 \\
\hline
\end{tabular}


Table 13: Masses and uncertainties for $N=17$ black hole (BH) stellar remnants hosted in binary systems. The post-merger body from GW 170817 is also included, where $-\Delta M=0.05=0.01$ (intrinsic error [2]) +0.04 (upper limit of ejected matter [4]). Masses are in solar units.

\begin{tabular}{lllll}
\hline \multicolumn{1}{c}{ system } & $M$ & $+\Delta M$ & $-\Delta M$ & ref \\
\hline A0620-00 & 6.61 & 0.23 & 0.17 & {$[44$} \\
Cyg X-1 & 10.05 & 3.20 & 3.20 & {$[74]$} \\
GRO J0422+32 & 3.97 & 0.95 & 0.95 & {$[40]$} \\
GRO J1655-40 & 6.30 & 0.27 & 0.27 & {$[74]$} \\
GRS 1915+105 & 14 & 4 & 4 & {$[46]$} \\
GS 1124-683 & 11.0 & 2.1 & 1.4 & {$[116]$} \\
GS 2000+25 & 7.46 & 0.32 & 0.32 & {$[74]$} \\
GS 2023+338 & 11.72 & 1.66 & 1.66 & {$[74]$} \\
H 1705-250 & 6.97 & 1.33 & 1.33 & {$[74]$} \\
LMC X-1 & 10.91 & 1.54 & 1.54 & {$[72$} \\
LMC X-3 & 7.55 & 1.62 & 1.62 & {$[74]$} \\
M33 X-7 & 15.65 & 1.45 & 1.45 & {$[71$} \\
NGC 3201 & 4.36 & 0.41 & 0.41 & {$[42$} \\
SAX J1819.3-2525 & 7.12 & 0.30 & 0.30 & {$[74]$} \\
XTE J1118+480 & 7.46 & 0.34 & 0.69 & {$[44$} \\
XTE J1550-564 & 9.10 & 0.61 & 0.61 & {$[73$} \\
4U 1543-47 & 9.42 & 0.97 & 0.97 & {$[74$} \\
GW 170817 & 2.74 & 0.04 & 0.05 & {$[2]$} \\
\hline
\end{tabular}

